\title{
Zur Methodik der Lößdifferenzierung auf der Grundlage der Carbonatverteilung
}

\author{
Von Friedhelm HäDrich, Freiburg i. Br. \\ Mit 10 Abbildungen und 4 Tabellen
}

\begin{abstract}
$\mathrm{Z}$ u s a m me $\mathrm{n}$ a s s ung. Im ersten Teil dieser Arbeit wird über eine Methode der geochemischen Carbonatfraktionierung in Gesamtcarbonat, Dolomit und Calcit und deren Fortentwicklung für die Untersuchung von Lößproben berichtet. Analysen- und Berechnungsfehler dieser und anderer herkömmlicher Methoden der Carbonatbestimmung werden diskutiert und Wege ihrer Vermeidbarkeit aufgezeigt.

Sodann werden Ergebnisse der Untersuchung von mehr als 300 Lößproben aus dem südlichen Oberrheingebiet dargestellt und statistisch ausgewertet. Nach den Dolomitgehalten lassen sich drei Lößaltersgruppen unterscheiden und weitgehend statistisch gegeneinander sichern. Die Dolomitgehalte sind innerhalb der einzelnen Lößpakete einheitlich hoch und ändern sich sprunghaft beim Ubergang von einem Löß in den anderen. Im Mittel liegt der Dolomitgehalt bei den Jüngeren Lössen bei 12,64\%, bei den Mittleren bei 10,91\% und bei den Alteren bei 5,53\%.

Die Dolomitverteilung in den Lößprofilen wird durch prälößgenetische Dolomitanreicherung in den Feinsand- und Schlufffraktionen der glazigenen und glazifluvialen Ablagerungen des Alpenvorlandes, als dem Herkunftsgebiet der Lösse erklärt.
\end{abstract}

$\mathrm{Su} \mathrm{m} \mathrm{m}$ a r y. The first part of this paper is concerned with a method of geochemical fractionating of carbonates and its further development for the investigation of loess samples.

This method allows to determine the amounts of total carbonate, dolomite and calcite. them.

Methodical mistakes of analysis and calculation are discussed and it is shown how to avoid

Finally the results of investigation of more than 300 loess samples from the southern Upper Rhine Valley are discribed and evaluated statistically.

According to the amounts of dolomite three loess groups of different ages could be distinguished. This distinction could be proved by statistical comparisons. In every single loess group the contents of dolomite are of the same level but by passing from one loess group into the other they change by leaps and bounds.

The average amount of dolomite of the younger loesses is about $12,64 \%$, of the loesses of medium ages about $10,91 \%$ and of the older ones about $5,53 \%$.

The dolomite distribution within the loess profiles is explained by preloessgenetical enrichment of dolomite in the fine sand and silt fractions of the glacial and glacifluvial deposits in the foreland of the Alps.

\section{Einführung}

Der Löß als pleistozänes äolisches Periglazialsediment ist in nahezu allen typischen Verbreitungsgebieten faziell deutlich gegliedert, so daß die Lößforscher zwar einerseits seinen Wert für eine chronostratigraphische Pleistozängliederung schon frühzeitig erkannten, andererseits diesen Wert oft weit überschätzten, falls nicht bestimmte Bedingungen (s. u.) vorlagen. So sind auch für das südliche Oberrheingebiet, das Gegenstand der vorliegenden Untersuchung ist, bis in die jüngste Vergangenheit hinein Arbeiten erschienen, die sich mit der stratigraphischen Gliederung, der Parallelisierung und der Datierung der Lösse und ihrer Paläoböden sowie mit deren geochemischen Eigenschaften beschäftigten (Guenther 1961, Bronger 1966, 1969, Bronger \& Hädrich 1969, Khodary-Eissa 1968, HäDRICH 1970). 
Eine gesicherte chronostratigraphische Gliederung der äolischen Pleistozänsedimente steht in Südwestdeutschland jedoch immer noch aus. Die Ursachen dafür müssen in erster Linie im Fehlen einer Reihe von "Fixpunkten“ und in ungünstigen stratigraphischen Bedingungen gesehen werden, die den eingangs angesprochenen Wert der oberrheinischen Lösse für die Pleistozängliederung einschränken. Als Argumente können in diesem $\mathrm{Zu}$ sammenhang angeführt werden:

1. Die Lösse lagern im südlichen Oberrheingebiet nicht auf datierten oder datierbaren Pleistozänterrassen, sondern aus tektonischen Gründen auf allen möglichen hier vorkommenden älteren Gesteinskomplexen. Die Ausnahme bildet lediglich der in Abschn. 4.4. dargestellte Fall.

2. Der Löß wurde während aller seiner Bildungsphasen in relativ hügeligem Gelände abgelagert, so daß die Gesamtmächtigkeiten örtlich stark schwanken und daß wir heute mit Hiaten und postgenetischen Profilverkürzungen zu rechnen haben, die auch die eingelagerten Paläoböden betreffen.

3. Die Lößforscher müssen sich wegen der umfangreichen anthropogenen Eingriffe auf Torsoprofile beschränken. Die Aufschlußverhältnisse werden zunehmend schlechter.

4. Die Lösse selbst - so roh und scheinbar unverändert sie auch vorliegen - sind hier nicht so reich gegliedert wie etwa in Hessen (Schönhals, Rohdenburg \& Semmel 1964; Semmel 1969, SEMmel et al. 1974) oder in Belgien (PAePe 1969).

5. Es fehlen weitgehend Artefakte und Petrefakte, mit Hilfe derer Zeitmarken gesetzt werden könnten.

6. Für eine Radiocarbondatierung fehlen i.d. R. humose Straten, bzw. einwandfreie Reste tierischer oder pflanzlicher Natur.

7. Die in rheinischen und hessischen Lössen (BrunnaCker 1971, Bibus 1973) auftretenden Tuffe fallen hier offensichtlich aus.

Aus all diesen Gründen ist es nicht verwunderlich, daß sich die Lößforscher gerade in diesem Raum immer mehr von der früher dominierenden profilmorphologischen Betrachtungsweise - ohne diese zu vernachlässigen - abkehren und verstärkt laboranalytischen Methoden aus der Bodenkunde, Sedimentologie und Geochemie zuwenden und zwar stets auf der Suche nach neuen, methodisch geeigneteren Wegen (Ḧ̈DRICH 1970).

In nahezu allen Lößarbeiten der letzten Jahrzehnte finden sich Daten zum „K a $1 \mathrm{k}$ g e h a $1 \mathrm{t}$ " von Lössen und Paläoböden, weil man schon frühzeitig seinen stratigraphischen Wert erkannte und in ihm einen wichtigen Gradmesser für postgenetische Materialveränderungen sah. Diese Arbeiten beschränken sich aber fast ausnahmslos auf die Beschreibung des Gesa m tcarbonats nach der vasvolumetrischen Scheibler-Methode, deren Ergebnisse von so vielen Zufälligkeiten abhängen und die bei Anwesenheit von D o l o m it schlicht als falsch bezeichnet werden müssen (vgl. Abschn. 2.3.1.).

Es hat aber in der Vergangenheit nicht an Versuchen gefehlt, neue, vorwiegend naßchemische Methoden zur Bestimmung von Gesamtcarbonat, vor allem aber seiner wichtigsten Fraktionen Calcit und Dolomit zu entwickeln, wobei man sich allerdings meist auf reine Kalk- und Dolomitgesteine beschränkte. Es sei hier beispielhaft nur auf die Arbeiten von Petersen \& Chesters (1966) und Nommix (1974) hingewiesen.

Im folgenden soll über eine zumindest für Lößproben neue Methode der geochemischen Carbonatfraktionierung berichtet werden, die erstmalig 1968 bei Proben aus Lößaufschlüssen von Bötzingen am Kaiserstuhl stichprobenweise angewandt worden war. Vorläufige Ergebnisse dieser ersten Untersuchungen finden sich bei KHODARY-EISSA (1968). Das Ergebnis war so erfolgversprechend, daß das Verfahren in den vergangenen Jahren unter ständiger methodischer Verbesserung bei der Untersuchung aller Proben aus Bötzingen a. K. und weiterer Aufschlüsse konsequent angewandt wurde. Das methodische Prinzip und einige anwendungstechnische Probleme wurden von HäDRICH bereits 1970 publiziert. 


\section{Darstellung und kritische Wertung der Methoden der Carbonatfraktionierung}

\subsection{Die Methodederkomplexometrischen Titration}

Es handelt sich um die Anwendung der von Müller (1964) nur für tonarme, d. h. reine und feste Carbonatgesteine empfohlenen Methode der komplexometrischen Titration ("Komplexometrie“) von $\mathrm{Ca}^{++}$und $\mathrm{Mg}^{++}$(im folgenden kurz $\mathrm{Ca}$ und $\mathrm{Mg}$ genannt) im salzsauren Extrakt auf Lößproben.

Nach ihm sollte diese Methode nur bei sehr reinen Carbonatgesteinen erlauben, den Dolomitgehalt zu berechnen und über den gleichzeitig ermittelten $\mathrm{CaCO}_{3}$-(Calcit-)Gehalt auch den Gesamtcarbonatgehalt zu bestimmen.

\subsubsection{Methodisches Prinzip:}

$0,5 \mathrm{~g}$ der gemahlenen, bei $105^{\circ} \mathrm{C}$ getrockneten Probe werden unter 8-minütigem Erhitzen $\left(100^{\circ} \mathrm{C}\right)$ zur Carbonatlösung mit $12,33 \%$ oiger $\mathrm{HCl}$ versetzt. Nach Zusatz von $\mathrm{NH}_{4} \mathrm{Cl}$, damit $\mathrm{Mg}$ in Lösung bleibt, und $\mathrm{NH}_{4} \mathrm{OH}$ zur Lösung werden die bei den nachfolgenden Titrationen störenden Schwermetallionen gefällt. Danach wird die Lösung in $500 \mathrm{ml}$ Meßkolben filtriert und aufgefüllt.

In je einem Aliquot des Filtrats wird mit dem bekannten, auf $\mathrm{Ca}$ und $\mathrm{Mg}$ spezifisch ansprechenden organischen Komplexbildner AeDTE in zwei getrennten Titrationsgängen (1. $\mathrm{Ca}+\mathrm{Mg}$, 2. Ca) unter Zusatz von Puffern und spezifischer Indikatoren durch Umrechnung über die jeweils verbrauchte AeDTE-Menge der Anteil von $\mathrm{Ca}$ und $\mathrm{Mg}$ an $100 \mathrm{~g}$ Probe bestimmt.

Wichtig ist, daß der erhaltene Mg-Anteil unter Heranziehung eines äquivalenten $\mathrm{Ca}-$ Anteiles auf $\mathrm{Dol}$ o m it mit einem Molverhältnis für $\mathrm{CaCO}_{3} \mathrm{zu} \mathrm{MgCO}_{3}$ wie $1: 1 \mathrm{um}$ gerechnet wird und der dabei verbleibende Rest an $\mathrm{Ca}$ auf $\mathrm{Calcit}$. Die auf diese Weise rechnerisch ermittelten Anteile an $\mathrm{CaCO}_{3}$ und $\mathrm{MgCO}_{3}$ ergeben zusammen das $\mathrm{G}$ e s a $\mathrm{m} \mathrm{t}$ $\mathrm{c}$ a r b on a ${ }^{1}$ ).

\subsubsection{Kritische Wertung der Methode der Komplexometrie}

Die Komplexometrie eignet sich besonders für Laboratorien mit bescheidener apparativer Ausstattung. Außer einer gewissen Grundausstattung mit Laborglaswaren und Chemikalien benötigt man lediglich einen Trockenschrank, einen Muffelofen (Zerstörung der organ. Substanz), eine Bürette (möglichst automatisch, aber nicht Bedingung) und ein heizbares Magnetrührwerk. Es ist sehr genau auf die Einhaltung konstanter Arbeitsbedingungen zu achten. Trotzdem sind subjektive Abweichungen in den Bedingungen nicht ganz vermeidbar, die als zufällige Fehler in die Bestimmung eingehen, weswegen die Reproduzierbarkeit der Ergebnisse nur als befriedigend bezeichnet werden kann. Gegenüber der im Abschn. 2.2. dargestellten Methode fallen die Werte für das Gesamtcarbonat um etwa $1,2-1,5 \%$, für den Dolomit um etwa $0,5 \%$ niedriger aus.

\subsection{Der HCl-Extrakt mit anschließender A A S-Messung}

Nicht zuletzt aus den in Abschn. 2.1.2. dargestellten Gründen wurde in den letzten Jahren bei allen untersuchten Proben parallel zur Komplexometrie der apparativ aufwendigere, arbeitstechnisch aber einfachere Weg über die Messung von $\mathrm{Ca}$ und $\mathrm{Mg}$ im Atomabsorptionsspekrophotometer (AAS) beschritten.

\subsubsection{Methodisches Prinzip}

Die $\mathrm{HCl} / \mathrm{AAS}-\mathrm{Methode} \mathrm{folgt} \mathrm{bis} \mathrm{zum} \mathrm{Auffüllen} \mathrm{des} \mathrm{Filtrats} \mathrm{auf} 500 \mathrm{ml}$ genau dem Vorgehen wie bei der Komplexometrie (Abschn. 2.1.). Lediglich die Titrationen wurden durch die schnellere AAS-Messung mit dem Gerät Perkin-Elmer 403 ersetzt. Für die Messung wurden Standard-Eichlösungen für $\mathrm{Ca}$ und $\mathrm{Mg}$ der Fa. Merck verwandt. Zum Aus-

1) Weitere methodische Details können jederzeit beim Verf. erfragt werden.

7 Eiszeitalter u. Gegenwart 
schalten eines Störioneneinflusses bei der Ca-Messung wurde $\mathrm{SrCl}_{2}$ zugesetzt. Die zur Messung kommenden Filtratmengen mußten 50 fach verdünnt werden. Die Berechnungen von Gesamtcarbonat und Dolomit erfolgten wie im Abschn. 2.1.1. angedeutet.

\subsubsection{Weitere methodische Änderungen}

Da bei der AAS-Messung Schwermetallionen nicht in dem Maße stören, wie bei der Komplexometrie, kann man auf die Fällung der Schwermetalle verzichten. Mit anderen Worten, man kann die Lösung ohne vorherigen $\mathrm{Zusatz}$ von $\mathrm{NH}_{4} \mathrm{Cl}$ und $\mathrm{NH}_{4} \mathrm{OH}$ filtrieren und das auf $500 \mathrm{ml}$ aufgefüllte Filtrat nach entsprechendem $\mathrm{SrCl}_{2}$-Zusatz und nach der Verdünnung zur Messung bringen.

Alle im Abschn. 4 dargestellten Daten entstammen noch aus der jahrelang geübten Behandlung und Messung nach Abschn. 2.2.1. Lediglich die $\mathrm{HCl} / \mathrm{AAS}-$ Werte in Abb. 2 (Proben von Eichstetten) wurden ohne Schwermetallfällung nach Abschn. 2.2.2. gewonnen.

\subsection{Die systematischen Fehlerder Methoden}

Es ist gleichgültig, welchen Weg der Carbonatfraktionierung - komplexometrische Titration oder $\mathrm{HCl} / \mathrm{AAS}-$ Methode mit oder ohne vorherige Schwermetallfällung - man beschreitet, es müssen in jedem Falle zweierlei Unsicherheiten in Kauf genommen werden, die sich als systematische Fehler in den Ergebnissen niederschlagen:

1. Fehler durch Abweichungen in der stöchiometrischen Zusammensetzung von Dolomit und Calcit

Der in der Probe vorliegende Dolomit weist nicht unbedingt das exakte Molverhältnis von $1: 1$ auf, weil ein Teil des $\mathrm{Mg}$ durch $\mathrm{Ca}, \mathrm{Fe}$ und $\mathrm{Mn}$ vertreten sein kann; es gibt aber auch Mg-haltige Calcite (MüLler 1964, 203 f). Dieser Fehler läßt sich bei den hinsichtlich ihrer Mineralvergesellschaftung äußerst heterogen zusammengesetzten Lössen wohl auch durch den Einsatz röntgenographischer Methoden (MüLLER 1964, 203 ff.) nicht exakt ermitteln und damit auch nicht vermeiden. Auf jeden Fall handelt es sich dabei um einen Fehler, der die Ergebnisse sowohl erhöhen als auch erniedrigen kann.

2. Fehler durch Einbeziehung silikatbürtiger Erdalkalien

Durch die 8 minütige Einwirkung der heißen $\mathrm{HCl}$ wird ein zunächst unbekannter, nach $\mathrm{Ab}$ schnitt 2.3.1. aber einzuschätzender Anteil von $\mathrm{Ca}$ und $\mathrm{Mg}$ aus silikatischer Bindung (Tonminerale und leicht verwitterbare Primärsilikate) freigesetzt und in die Bestimmung einbezogen, so daß die errechneten Gesamtcarbonat- und Dolomitwerte gegenüber den entsprechenden realen Gehalten immer zu hoch ausfallen müssen.

2.3.1. Fehlereinschätzung durch Vergleich mit der Methode der konduktometrischen Gesamtcarbonatbestimmung

Um wenigstens den zweiten, im vorhergehenden Abschnitt erwähnten Fehler einschätzen zu können, bedarf es des Vergleichs der Daten aus der Komplexometrie oder aus der $\mathrm{HCl}$ /AAS-Messung mit einer anderen, genaueren Methode der Gesamtcarbonbestimmung.

Für diesen Vergleich erwies sich die konduktometrische Methode der Gesamtcarbonatbestimmung mittels heißer Ortho-Phosphorsäure und zugeschaltetem Gasanalysegerät der Fa. W öst h off / Bochum (Schlichting \& Blume 1966, 108) als besonders geeignet, weil die Reaktion im Sinne einer vollständigen Carbonatlösung verläuft, da sich das schwersösliche Ca-Phosphat bildet und weil störende Gase durch entsprechende vorgeschaltete Absorber eliminiert werden können.

Die „W östh off-Methode“ liefert aber nur dann zuverlässige Gesamtcarbonatwerte, wenn die Proben ausschließlich $\mathrm{CaCO}_{3}$ und keine anderen Carbonatformen enthalten. Ahnlich wie bei der gasvolumetrischen Gesamtcarbonatbestimmung nach ScHEIBLER werden auch bei der W ös th of $\mathrm{f}$ - Methode die erhaltenen Meßwerte i. d. R. auf $\mathrm{CaCO}_{3}$ umgerechnet und zwar unter Vernachlässigung der möglicherweise vorhandenen $\mathrm{MgCO}_{3}-$ Anteile aus den dolomitischen Bestandteilen der Proben. 
Bei Anwesenheit von Dolomit schleicht sich durch diese Berechnungsweise ein ausnahmslos positiver systematischer Fehler in die Daten ein, der mit dem Dolomitgehalt in seiner Größenordnung ansteigt und der nur beseitigt werden kann, wenn der Dolomitgehalt, z. B. aus der Bestimmung nach Abschn. 2.1. oder 2.2. bekannt ist.

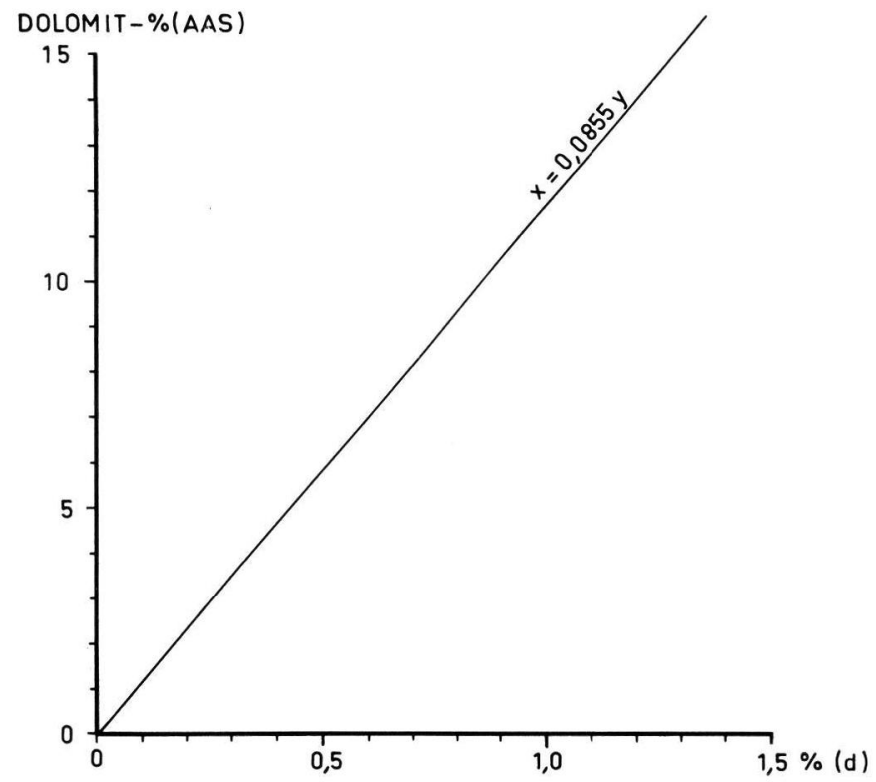

Abb. 1. Differenzwerte (d) zur Korrektur des Gesamtcarbonats (Wösthoff) als Funktion des Dolomitgehaltes ( $\mathrm{d}=$ Dolomit- $\%$ x 0,0855) ${ }^{2}$ )

Die Gesamtcarbonatwerte nach Wösth off bedürfen daher eines negativen Korrekturfaktors (d), der sich, wie aus $\mathrm{Abb} .1 \mathrm{zu}$ entnehmen ist, bei den untersuchten Lössen $z$ wischen 0,2 und $1,2 \%$ bewegt und allgemein aus der linearen Beziehung $x=0,0855 \mathrm{y}$ $(\mathrm{x}=\mathrm{d})$ berechnet werden kann. Dieser Korrekturfaktor resultiert aus rein stöchiometrischen Überlegungen und ist daher unabhängig von der Art der Probe und kann auch für höhere Dolomitgehalte berechnet bzw. extrapoliert werden. Dieser Faktor ist in seiner Genauigkeit lediglich abhängig von der Genauigkeit der Dolomitbestimmung nach Abschn. 2.1. oder 2.2.

Das reale Ges a m t c a r b o n a t berechnet sich demnach wie folgt:

$$
\text { Gesamtcarbonat }=\text { Gesamtcarbonat (W ös th off })-d
$$

Zur Bestimmung der Dolomitgehalte und realer Gesamtcarbonatwerte bedarf es daher der kombinierten Anwendung der HCl/AAS-Methode (Abschn. 2.2.) und der Konduktometrie mit nachfolgender Korrektur.

Die Abb. 2 veranschaulicht beispielhaft die Beziehung zwischen den korrigierten Gesamtcarbonatwerten aus der Konduktometrie mit dem W ös th of f-Gerät und denen nach der $\mathrm{HCl} / \mathrm{AAS}-\mathrm{Methode}$ für 50 dolomitreiche Lößproben von Eichstetten a. $\mathrm{K}$.

2) Dolomit- $\%$ bedeutet Dolomitgehalt in $\%$. 


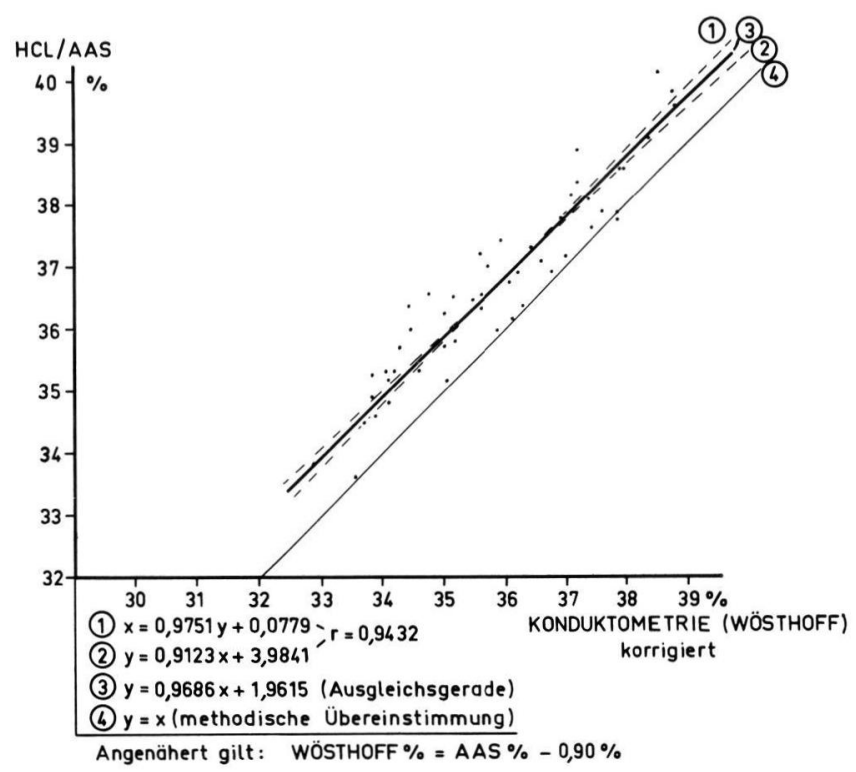

Abb. 2. Gesamtcarbonat im Methodenvergleich Eichstetten L1+L2 $(\mathrm{N}=50)$.

Wichtig für die Einschätzung beider Methoden ist der Abstand der Ausgleichsgeraden 3 von der Geraden 4 (angenommene methodische Übereinstimmung). Werte.

Die $\mathrm{HCl} / \mathrm{AAS}$-Werte liegen im Mittel um 0,90\% höher als die realen W ö s th of f-

Es ist naheliegend, diesen Überschuß bei der $\mathrm{HCl} / \mathrm{AAS}-\mathrm{Methode}$ aus der Einbeziehung silikatbürtiger Erdalkalien zu interpretieren.

\subsection{Methodischer Ausblick}

$\mathrm{Zu}$ gesicherten Aussagen über die Carbonatverteilung und Carbonatfraktionierung in Lößproben gelangt man m. E. nur durch den Einsatz von mindestens zwei voneinander unabhängigen Methoden, deren Ergebnisse bzw. Aussagen sich wechselseitig zur Fehlerreduktion verwenden lassen. Die Gesamtcarbonatwerte nach Wös th of $f$ lassen sich über die Dolomitgehalte aus der HCl/AAS-Methode präzisieren (Abb. 1) und durch Vergleich dieser korrigierten Wö s th of $\mathrm{f}$ - Werte mit den Gesamtcarbonatgehalten aus der $\mathrm{HCl}$ /AAS-Methode läßt sich der Fehler abschätzen, der durch Einbeziehung von silikatbürtigen Erdalkalien in das $\mathrm{HCl} / \mathrm{AAS}-\mathrm{Gesamtcarbonat}$ eingeht (Abb. 2).

In der Abb. 3 ist zur besseren Verdeutlichung der Ausführungen der von mir gewählte Analysengang schematisch dargestellt.

$\mathrm{Da}$ der silikatbürtige „Gesamtcarbonatüberschuß" bei der $\mathrm{HCl}$ /AAS-Methode mit $0,90 \%$ im Mittel nur einem Dolomitüberschuß von 0,29\% entspricht und dieser Fehler bei allen Lößproben gleichsinnig auftreten dürfte, kann m. E. die Methode der Carbonatfraktionierung zur Charakterisierung und Differenzierung von Lössen gleicher oder ähnlicher Zusammensetzung herangezogen werden.

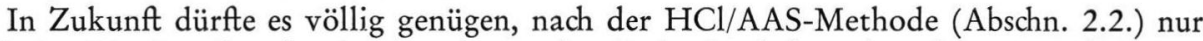
noch den Mg-Anteil zu bestimmen, daraus den Dolomitgehalt zu berechnen, parallel dazu den Gesamtcarbonatgehalt nach W östh off zu erfassen und diesen entsprechend dem Dolomitgehalt zu korrigieren. 


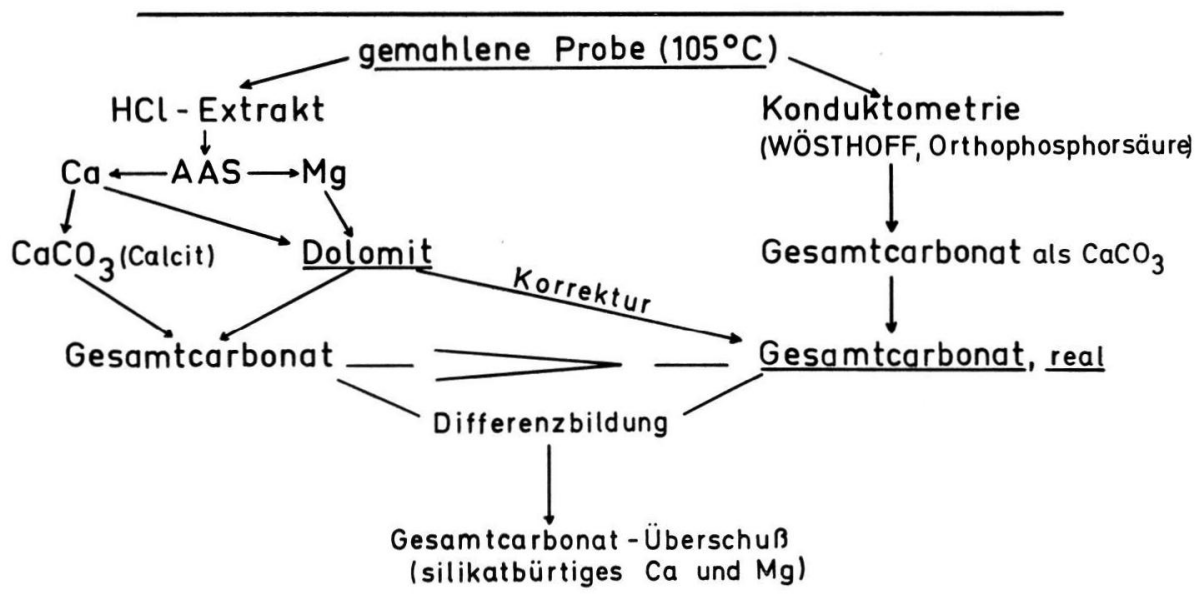

Abb. 3. Carbonatfraktionierung bei Lössen, Analysengang und Berechnungsschema.

Wenn auch gewisse Unsicherheiten im geschilderten Analysengang den Sedimentologen und Petrographen noch skeptisch stimmen, weil die Berechnung der Dolomitgehalte nach Abschn. 2.3. vielleicht als fragwürdig erscheinen mag, den Lößstratigraphen sollte dies wenig kümmern. Ihm kann es gleichgültig sein, ob eine bestimmte Lößschicht nur 12,3\% statt der bestimmten 12,5\% Dolomit enthält, wenn nur dieser Dolomitgehalt sich als relativ konstantes Merkmal über den gesamten als genetisch einheitlich angesehenen Lößkomplex erstreckt und sich deutlich vom nächsten über oder unter ihm abhebt. Damit wird der Dolomitgehalt zum Charakteristikum dieses Komplexes.

Wenn im folgenden von Gesamtcarbonat die Rede ist, dann handelt es sich ausschließlich um Daten aus der HCl/AAS-Methode nach Abschn. 2.2.1. Das gilt auch für die Proben aus Eichstetten. Die späteren Parallelbestimmungen nach Abschn. 2.2.2. und nach Wösth off (vgl. Abb. 2) konnten nicht weiter ausgewertet werden. Es ist verständlich, daß für die lithostratigraphischen Aussagen, wie sie in Abschn. 4 getroffen werden, nur methodisch einheitlich behandelte Daten herangezogen werden konnten.

\section{Die Untersuchungsobjekte}

Seit 1968 wurden mehr als 300 Lößproben unterschiedlichen Alters aus insgesamt 7 Aufschlüssen des Kaiserstuhls und der Lahr-Emmendinger Vorbergzone des Schwarzwaldes (Abb. 4) auf Gesamtcarbonat- und Dolomitgehalt untersucht ${ }^{3}$ ).

Unter ihnen befinden sich auch einige Stichproben aus C-Horizonten rezenter LößParabraunerden aus der Emmendinger Vorbergzone (HäDRICH 1966) und der Teninger Fläche in der nördlichen Freiburger Bucht (vgl. dazu Abschnitt 4.4).

Die bearbeiteten Lößprofile sind in Abb. 5 stark schematisiert dargestellt. Sie unterscheiden sich nach Anzahl und damit Alter sowie Mächtigkeit der Lösse sehr stark. Teilweise sind sie bereits aus der Literatur bekannt, so die von Bötzingen a. K. durch KHODARY-EISSA (1968) und die von Riegel a. K. - bis auf einige Abweichungen - durch GuENTHER (1961).

3) Die Untersuchungen im Labor wurden ausgeführt von den techn. Assistentinnen L. Henninger, B. Rieser, B. Röttges, K. v. RuepPrecht und H. SCHLEnKER, denen ich an dieser Stelle herzlich danken möchte. 

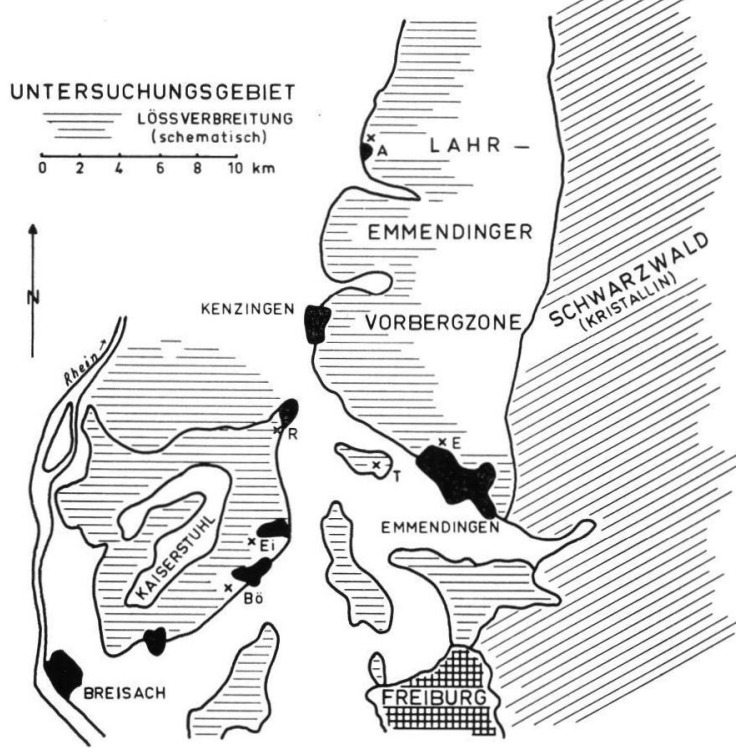

Abb. 4. Die Verbreitung des Lösses im Untersuchungsgebiet.

\section{EICHSTETTEN BÖTZINGEN a.K. EMMENDINGEN ALTDORF}

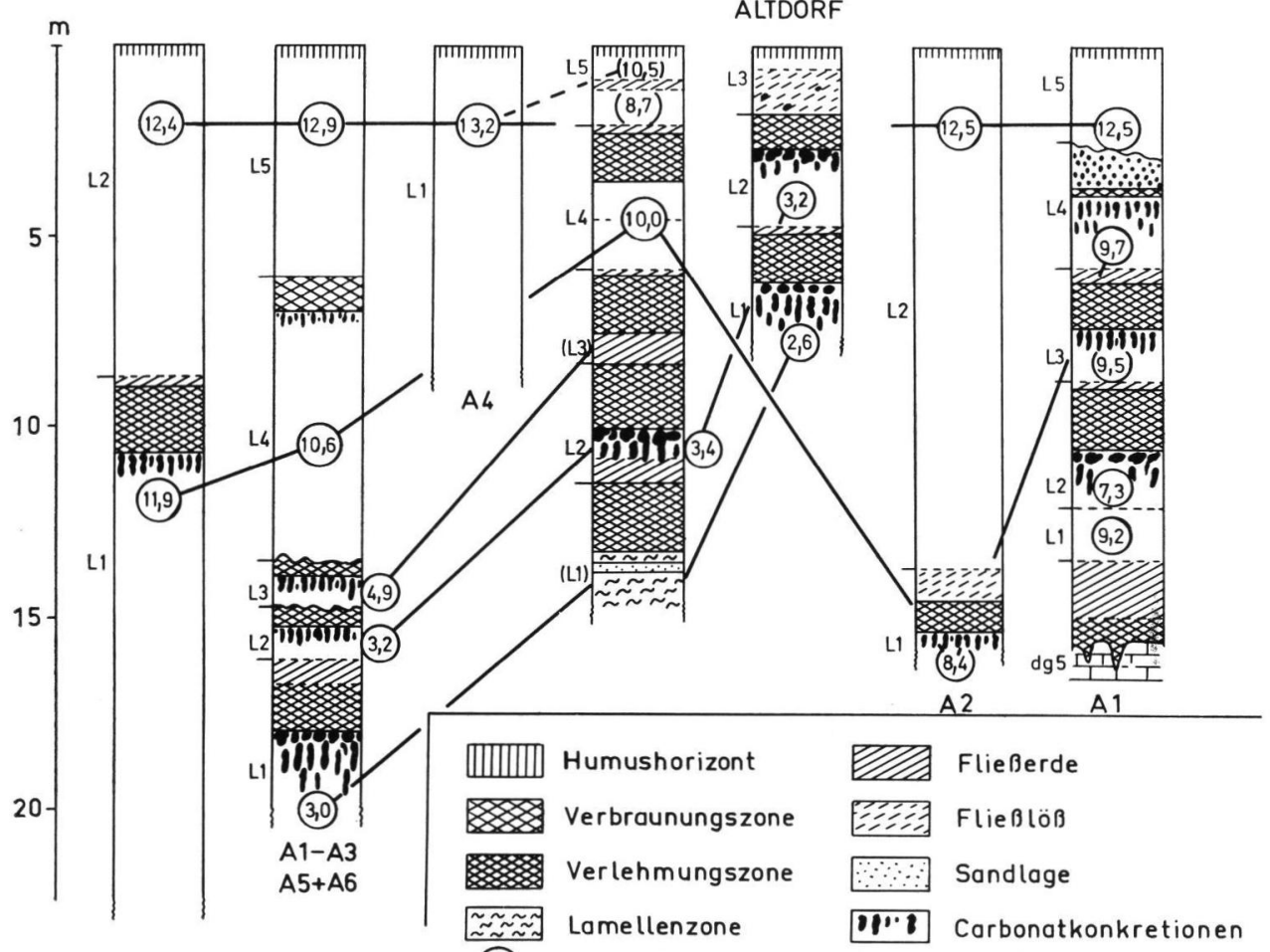

(12,5) Dolomitgehalt in \% (Mittelwert)

Abb. 5. Vergleichende Stratigraphie oberrheinischer Lößaufschlüsse in vereinfachter Darstellung. 
Altdorf und Emmendingen wurden m. W. bisher noch nicht bearbeitet. Während in Bötzingen und Riegel die jüngeren Lösse dominieren, sind Altdorf und Emmendingen durch das Vorherrschen der älteren Lösse und der zwischengeschalteten mächtigen Paläoböden gekennzeichnet.

Bei Eichstetten a. K. handelte es sich um eine Bohrung, die von Mitarbeitern ${ }^{4}$ ) des Lehrstuhls für Geographie und Hydrologie des Geographischen Instituts I der Universität Freiburg i. Br. im Sommer 1974 im Zusammenhang mit einem hydrologischen Projekt bis in eine Tiefe von fast $23 \mathrm{~m}$ niedergebracht wurde und an der ich mich beteiligen durfte.

Die Lösse sind bis auf eine Ausnahme bei Bötzingen A 4 jeweils durch Paläobodenreste vom Typus der Parabraunerde mit stark entwickelten Carbonatkonkretions-Horizonten an ihrer Basis gegliedert bzw. unterlagert, sie selbst sind carbonatreich (20-40\%), tonarm $(<15 \%)$ und extrem humusarm (i.d.R. $\langle 0,6 \%)$.

Stark durch pedogenes Material „verunreinigte“ Profilabschnitte (Fließlösse oder Fließerden) wurden von der Untersuchung und Auswertung ausgenommen.

$\mathrm{Da}$ es - allein schon aus den in Abschn. 1 angeführten Gründen - nicht Sinn und Zweck dieser Arbeit ist, die Chronostratigriphie der oberrheinischen Lösse an diesen teilweise recht komplizierten Profilen neu aufzurollen, müssen Ausführungen über stratigraphische Details entfallen.

Wenn an einer Lokalität mehrere Aufschlüsse bearbeitet wurden, dann wurden diese mit A 1 bis A n durchnumeriert. Die Proben wurden, mit Ausnahme der Bohrung Eichstetten, in Abständen von je $20 \mathrm{~cm}$ entnommen. Bei Eichstetten war es im Durchschnitt ein Abstand von $37 \mathrm{~cm}$. Die Lösse wurden in jedem Profil von unten nach oben (genetisches Prinzip) schematisch durchnumeriert und mit L 1 bis $\mathrm{L} n$ bezeichnet. Alle Bestimmungen wurden im Laufe der letzten Jahre bis zu viermal wiederholt. Doppelbestimmungen liegen bei allen Proben vor. Die nicht die Carbonfraktionen betreffenden pedologischen Parameter von Lössen und Paläoböden können im Rahmen dieser Arbeit leider keine Berücksichtigung finden.

Im Rahmen eines von der UNESCO/Paris finanziell unterstützten Untersuchungsprogrammes zur Vereinheitlichung der Ansprache von pleistozänen und holozänen Sedimenten, das dankenswerterweise J. FINK/Wien initiiert und organisiert hat, sind in den letzten eineinhalb Jahren mehr als 100 Proben von Lössen und verwandten Sedimenten aus zahlreichen europäischen Ländern analysiert worden. Diese Untersuchungen sind noch nicht abgeschlossen; daher können die Ergebnisse der Carbonatfraktionierung an dieser Stelle noch nicht mitgeteilt werden.

\section{Die Ergebnisse der bisherigen Untersuchungen}

\subsection{Das Gesamtcarbonat}

Ergebnisse über Gesamtcarbonatbestimmungen - gleich, welcher methodische Weg auch beschritten wurde - liegen aus dem südlichen Oberrheingebiet in großer Zahl vor. Ich verweise hier nur auf die Arbeiten von Bronger (1966, 1969), Khodary-Eissa (1968) und HäDRICH (1970). Aus allen diesen Untersuchungen geht hervor, daß ihr stratigraphischer Wert, bzw. ihre Bedeutung für eine relative Chronologie der Lösse sehr gering ist (vgl. auch Abb. 10).

Die Gesamtcarbonatgehalte ergaben sich bei meinen Bestimmungen und Berechnungen zwangsläufig, und so sollen sie dem Leser nicht vorenthalten bleiben, zumal es nicht un-

4) Den Herren G. Luft und G. Morgenschweis möchte ich an dieser Stelle herzlich danken. 
interessant ist, von den einzelnen Lössen den Anteil der Carbonatfraktionen am Gesamtcarbonat zu kennen (Tab. 2).
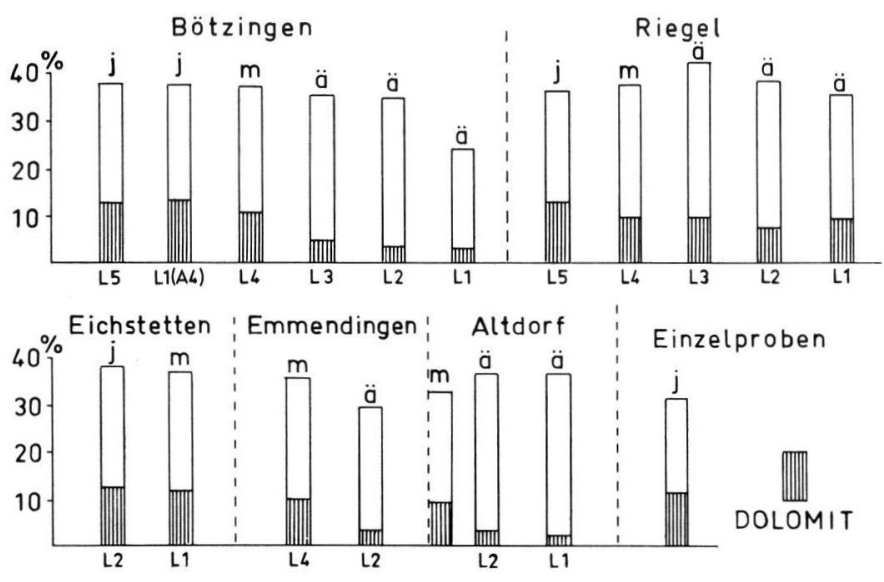

$\mathrm{j}=$ jüngerer Löss, $m=$ mittlerer Löss, $\ddot{a}=$ älterer Löss

Abb. 6. Mittlere Gesamtcarbonat- und Dolomitgehalte der Lösse.

Die Abbildung 6 der mittleren Gesamtcarbonat- und Dolomitgehalte zeigt, daß das Gesamtcarbonat von Profil zu Profil und von Löß zu Löß innerhalb der Profile vergleichsweise nur geringen Schwankungen unterworfen ist. Im Mittel aller Proben liegt der Gesamtcarbonatgehalt bei den sogenannten Jüngeren Lössen bei 37,1\% (HCL/AAS), bzw. bei 36,2\% (korrigiert ${ }^{5}$ ), real) $(\mathrm{n}=167$ ), bei den Mittleren Lössen bei 36,9\% real bei 36,0\% $(n-83)$ und bei den Alteren Lössen bei $34,3 \%$, bzw. real bei 33,4\% $(\mathrm{n}=51)$. Demnach ist eine schwache Tendenz der Abnahme des Gesamtcarbonats mit zunehmendem Alter des Lösses gegeben. Diese Tendenz wird vor allem durch die beiden Lösse L 1 von Bötzingen und L 2 von Emmendingen gesteuert. Nach dem Gesamtcarbonatgehalt ist keine Differenzierung und Parallelisierung der Aufschlüsse möglich, da sich die Unterschiede in den jeweiligen Mittelwerten nur selten statistisch sichern ließen. Ganz andere Aspekte eröffnen sich, wenn die Dolomitfraktion für eine Gliederung von Profilen herangezogen wird.

\subsection{Die Dolomitfraktion}

Die Dolomitgehalte zeigen über alle untersuchten Lößprofile hinweg einen charakteristischen Trend der Abnahme von oben nach unten, von jung nach alt. Diese Abnahme erfolgt nicht stetig innerhalb der Lösse, sondern sprunghaft an den Substratgrenzen, den Paläoböden. Darin wird die kausale Verknüpfung des Dolomitgehaltes mit dem Lößalter bzw. der Lößgenese deutlich (vgl. Abschn. 5.4.2.).

Zur Verdeutlichung der Homogenität der Lösse hinsichtlich ihres Dolomitgehaltes wurde in Tabelle 1 u. a. der Dolomitgehalt des Jüngeren Lösses L 2 des Aufschlusses A 2 von Riegel a. K. beispielhaft dargestellt.

5) Unter Zugrundelegung der durchschnittlichen Ergebnisse aus Abb. 2 (vgl. Abschn. 2.3.1.). 
Tabelle 1

Dolomit- und Gesamtcarbonatgehalt beim Jüngeren Löß L 2 vom Aufschluß Riegel A 2

\begin{tabular}{|c|c|c|c|}
\hline $\begin{array}{l}\text { Tiefe } \\
\mathrm{cm}\end{array}$ & $\begin{array}{c}\text { Gesamt- } \\
\text { carbonat } \\
\mathbf{0} \%\end{array}$ & $\begin{array}{c}\text { Dolomit } \\
0 / 0\end{array}$ & $\begin{array}{l}\text { Dolomit } \\
\text { in } \% / 0 \mathrm{v} \text {. } \\
\text { Ges.-Carb. }\end{array}$ \\
\hline $60-80$ & 34,88 & 12,10 & 34,69 \\
\hline-100 & 36,17 & 12,31 & 34,02 \\
\hline-120 & 36,28 & 12,16 & 33,52 \\
\hline-140 & 37,00 & 12,21 & 33,00 \\
\hline-160 & 37,91 & 12,72 & 33,55 \\
\hline-180 & 38,57 & 11,99 & 31,09 \\
\hline-200 & 43,72 & 12,59 & 28,80 \\
\hline-220 & 43,34 & 12,97 & 29,93 \\
\hline-240 & 40,38 & 12,78 & 31,65 \\
\hline-260 & 40,49 & 13,45 & 33,22 \\
\hline-280 & 40,20 & 12,65 & 31,47 \\
\hline-300 & 37,99 & 12,61 & 33,19 \\
\hline-320 & 37,31 & 12,52 & 33,56 \\
\hline-340 & 37,78 & 13,26 & 35,10 \\
\hline-360 & 35,05 & 11,89 & 33,92 \\
\hline-380 & 34,94 & 11,42 & 32,68 \\
\hline-400 & 36,76 & 13,29 & 36,15 \\
\hline-420 & 37,88 & 13,46 & 35,53 \\
\hline-440 & 37,55 & 13,29 & 35,39 \\
\hline-460 & 38,71 & 14,85 & 38,36 \\
\hline-480 & 37,25 & 12,65 & 33,96 \\
\hline-500 & 37,03 & 13,10 & 35,38 \\
\hline-520 & 37,43 & 13,16 & 35,16 \\
\hline-540 & 35,72 & 11,95 & 33,45 \\
\hline-560 & 36,44 & 13,07 & 35,87 \\
\hline-580 & 35,25 & 12,00 & 34,04 \\
\hline-600 & 36,20 & 11,83 & 32,68 \\
\hline-620 & 37,20 & 13,46 & 36,18 \\
\hline-640 & 35,78 & 11,61 & 32,45 \\
\hline-660 & 35,71 & 12,40 & 34,72 \\
\hline-680 & 35,82 & 12,80 & 35,73 \\
\hline-700 & 35,24 & 12,13 & 43,42 \\
\hline-720 & 34,89 & 10,75 & 30,81 \\
\hline-740 & 34,53 & 10,34 & 29,95 \\
\hline-760 & 35,41 & 10,79 & 30,47 \\
\hline-780 & 36,06 & 12,59 & 34,91 \\
\hline-800 & 36,41 & 11,74 & 32,24 \\
\hline
\end{tabular}

\begin{tabular}{|c|c|c|c|}
\hline $\begin{array}{l}\text { Tiefe } \\
\mathrm{cm}\end{array}$ & $\begin{array}{c}\text { Gesamt- } \\
\text { carbonat } \\
0 \%\end{array}$ & $\begin{array}{c}\text { Dolomit } \\
\mathbf{0} \%\end{array}$ & $\begin{array}{l}\text { Dolomit } \\
\text { in } \% \mathrm{v} \text {. } \\
\text { Ges.-Carb. }\end{array}$ \\
\hline $800-820$ & 36,54 & 12,72 & 34,81 \\
\hline-840 & 36,00 & 11,83 & 32,86 \\
\hline-860 & 35,46 & 11,45 & 32,29 \\
\hline-880 & 35,29 & 11,47 & 32,50 \\
\hline-900 & 36,34 & 11,59 & 31,89 \\
\hline-920 & 36,01 & 11,57 & 32,13 \\
\hline-940 & 38,14 & 13,33 & 34,95 \\
\hline-960 & 37,27 & 12,25 & 32,87 \\
\hline-980 & 38,38 & 13,75 & 35,83 \\
\hline-1000 & 39,14 & 13,88 & 35,46 \\
\hline-1020 & 38,33 & 13,10 & 34,18 \\
\hline-1040 & 37,75 & 13,73 & 36,37 \\
\hline-1060 & 38,85 & 14,07 & 36,22 \\
\hline-1080 & 37,86 & 13,43 & 35,47 \\
\hline-1100 & 37,52 & 12,42 & 33,10 \\
\hline-1120 & 34,88 & 11,83 & 33,92 \\
\hline-1140 & 35,69 & 11,81 & 33,09 \\
\hline-1160 & 34,03 & 11,61 & 34,12 \\
\hline-1180 & 34,23 & 11,54 & 33,71 \\
\hline-1200 & 33,31 & 11,58 & 34,76 \\
\hline-1220 & 32,91 & 11,61 & 35,28 \\
\hline-1240 & 33,14 & 11,57 & 34,91 \\
\hline-1260 & 32,81 & 10,86 & 33,10 \\
\hline-1280 & 34,36 & 12,71 & 36,99 \\
\hline-1300 & 34,51 & 12,48 & 36,16 \\
\hline-1320 & 36,58 & 13,46 & 36,80 \\
\hline-1340 & 35,63 & 12,21 & 34,27 \\
\hline-1360 & 36,05 & 13,56 & 37,61 \\
\hline-1380 & 35,80 & 13,67 & 38,18 \\
\hline-1400 & 35,93 & 13,77 & 38,32 \\
\hline-1420 & 35,41 & 13,10 & 37,00 \\
\hline-1440 & 35,72 & 12,42 & 34,77 \\
\hline-1460 & 41,64 & 12,14 & 29,15 \\
\hline
\end{tabular}

Alle Werte entstammen der AAS-Messung nach Abschnitt 2.2.1.

Besonders klar zeigt sich der o. g. Trend des sprunghaften Verhaltens von Löß zu Löß an den Aufschlüssen von Bötzingen a. K. Hier nimmt der Dolomitgehalt von ca. $13 \%$ in den Jüngeren Lössen über $10,6 \%$ bei den Mittleren auf 3,0\% bei den Ailteren ab.

Dieses Verteilungsmuster findet sich in etwa bei allen Aufschlüssen wieder. Die Begriffe "Jünger“, "Mittel“ und „Alter“ sind nicht unbedingt identisch mit den Begriffen Jung-, Mittel- und Altpleistozän, sie orientieren sich neben der stratigraphischen Position vor allem an den Lößeigenschaften und hier besonders am Dolomitgehalt. Die Jüngeren Lösse sind in den vollständigen Aufschlüssen jeweils zuoberst gelegen und weisen den höchsten Dolomitgehalt auf. Es handelt sich immer nur um e in Lößpaket. Dieses ist durch den ersten markanten fossilen Boden vom Mittleren Löß getrennt, der in seinen Eigenschaften noch sehr dem Jüngeren ähnelt, im Dolomitgehalt und in der stratigraphischen Position aber an zweiter Stelle steht. Darunter beginnt - wiederum durch einen kräftigen Paläoboden getrennt und in sich durch Paläoböden gegliedert - eine Folge von Älteren Lössen, die verhältnismäßig einheitlich sind, sich aber i.d.R. sehr deutlich - vor allem im Dolomitgehalt — vom hangenden Mittleren Löß abheben. 
Um eine verbindliche Aussage über die Homogenität der einzelnen Lößkomplexe bzw. deren Unterscheidbarkeit treffen zu können, muß die Statistik bemüht werden ${ }^{6}$ ). Die zentrale Frage war: Sind die Unterschiede in den Mittelwerten zwischen den einzelnen Lößkomplexen statistisch zu sichern?

\subsection{Statistische Berechnungen}

\subsubsection{Statistik der Dolomitfraktion}

Anhand der Übersicht über die statistischen Maßzahlen (Tab. 2) sollen die einzelnen Lösse noch etwas näher vorgestellt werden. Die Lösse wurden hier nach ihrem Alter geordnet.

Tabelle 2

Ubersicht über die statistischen Maßzahlen für die untersuchten Lösse.

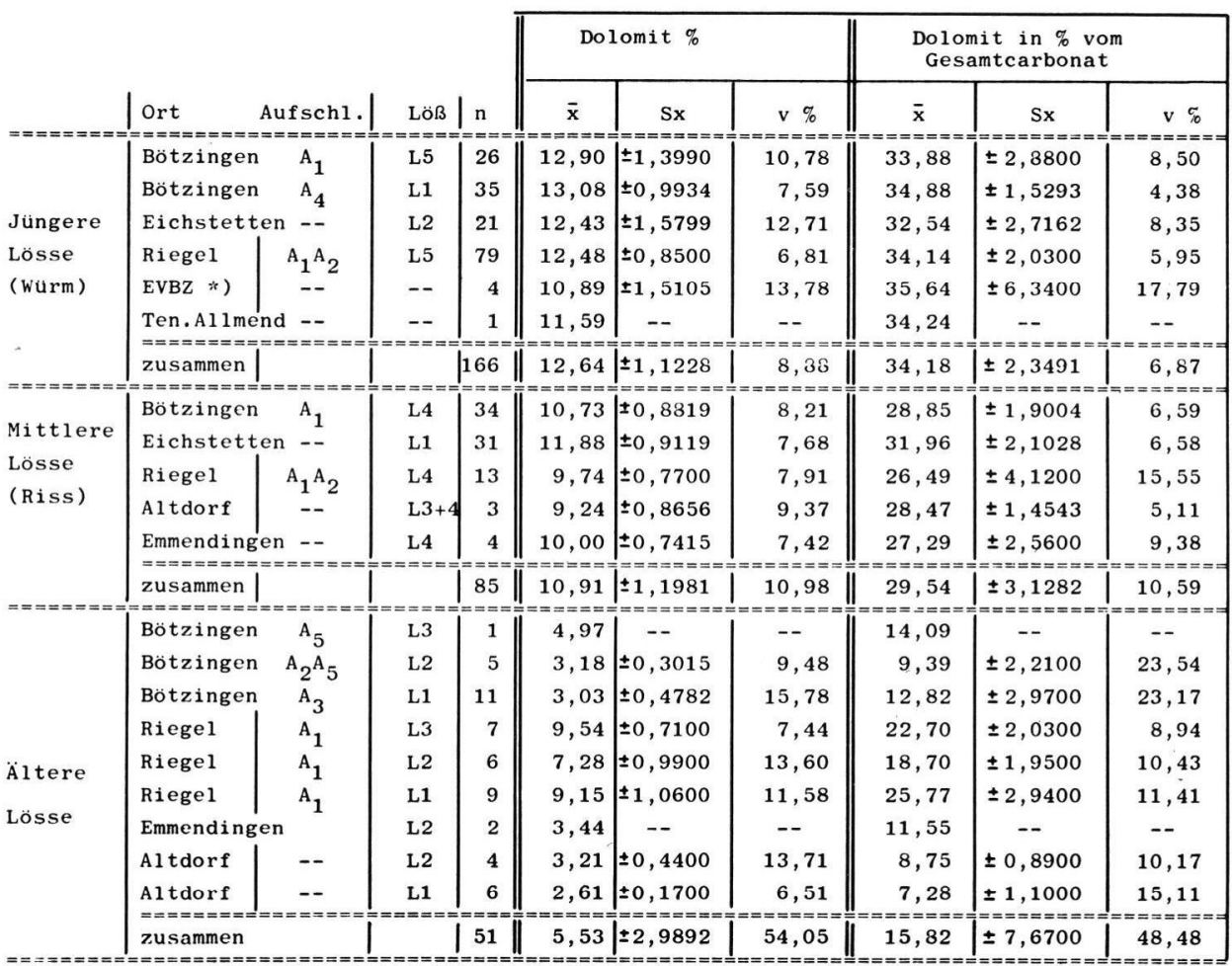

*) Einzelproben aus der Emmendinger Vorbergzone

Maßgebend für diese Gliederung waren der Dolomitgehalt und die stratigraphische Position in den einzelnen Aufschlüssen. Durch Vergleich der Daten über Mittelwert $(\overline{\mathrm{x}})$, Streuung $(\mathrm{Sx})$ und Variationskoeffizient $(\mathrm{v} \%)$ innerhalb der senkrechten Spalten können Gemeinsamkeiten und Unterschiede auf einen Blick erfaßt werden.

Auffallend ist die besonders große Homogenität der Daten für die Jünge ren $\mathrm{L}$ ö s se, die sich in der geringen Streuung und im niedrigen Variationskoeffizienten ausdrückt, was besonders für die statistische Zusammenfassung aller jüngeren Lösse gilt.

6) Für zahlreiche wertvolle Anregungen bei der statistischen Auswertung des Datenmaterials danke ich Herrn Dipl.-Forstwirt K. KeILEN. 
Die Mittleren Lösse zeigen zwar im einzelnen sehr geringe Streuungsbetràge und Variationskoeffizienten. Diese Werte steigen aber bei statistischer Zusammenfassung beträchtlich. Dies gibt einen Hinweis auf die vergleichsweise größeren stofflichen Unterschiede innerhalb des Gesamtkollektivs der Mittleren Lösse.

Die relativ hohen Streuungswerte und Variationskoeffizienten der A $1 \mathrm{t}$ e r e n L ös se, insbesondere bei deren statistischer Zusammenfassung, wird hauptsächlich durch die Riegeler Lösse L1-L3 verursacht. Hierin zeigt sich deutlich die vorerst nicht zu klärende Sonderstellung dieser drei Lösse. Sie hätten nach den Ergebnissen der Carbonatfraktionierung eigentlich eher zu den Mittleren als zu den Älteren Lössen gestellt werden müssen. Dem stand allerdings ihre stratigraphische Position entgegen. Erst bei der Untersuchung eines noch größeren Stichprobenumfanges aus den Mittleren und Alteren Lössen wird man die Stellung der Riegeler Lösse L1-L3 vielleicht klären bzw. feststellen können, ob nicht die Alteren Lösse in sich noch weiter differenziert werden müssen. Das Verhalten dieser Alteren Riegeler Lösse zeigt sich auch in den Abbildungen 7-10. Abb. 7 bringt die Häufigkeitsverteilungen der Dolomitgehalte für die drei Lößaltersgruppen. Eindrucksvoll erscheint der Trend der Dolomitabnahme mit zunehmendem Lößalter.

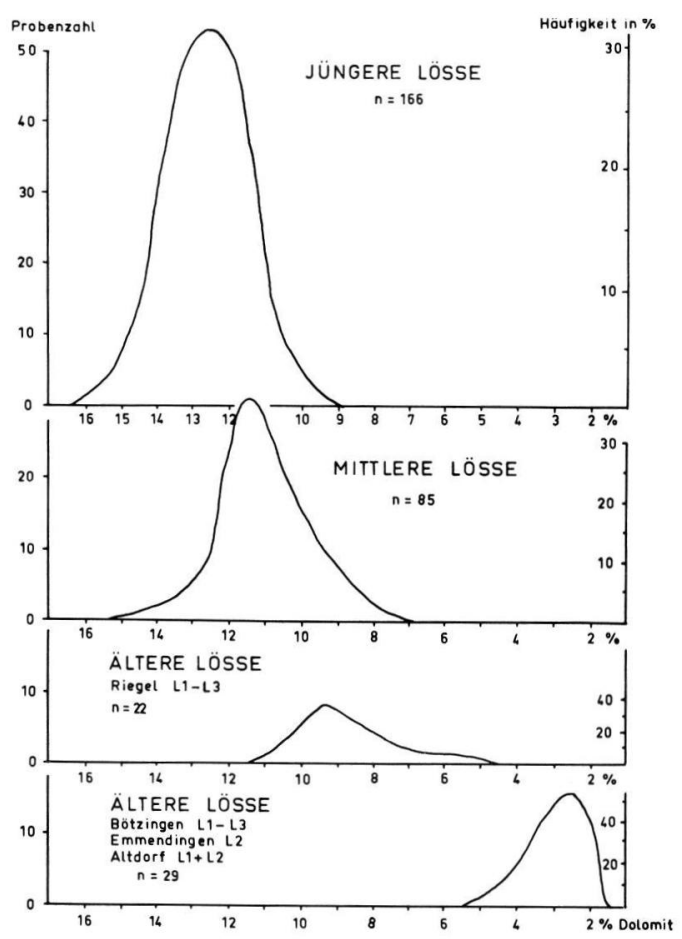

Abb. 7. Häufigkeitsverteilung des Dolomitgehaltes für die Lösse.

Um nun aber festzustellen, inwieweit sich die Lösse statistisch gegeneinander abgrenzen lassen, wurden die Unterschiede in den Mittelwerten zunächst für die Lösse innerhalb der Aufschlüsse einem t-Test unterzogen (Tab. 3). Wir erkennen, daß sich die Mittelwerte innerhalb der Aufschlüsse i.d.R. signifikant unterscheiden. Theoretisch sollte Nichtsignifikanz bei gleichaltrigen, Signifikanz bei verschiedenaltrigen Lössen herrschen. Aus Tab. 3 ist jedoch nicht so ohne weiteres zu entnehmen, ob die nichtsignifikanten Beziehungen in jedem Fall gleichaltrige Lösse betrifft. 
Tabelle 3

Vergleich der Mittelwerte aller Lösse im Signifikanztest (gegliedert nach Aufschlüssen).

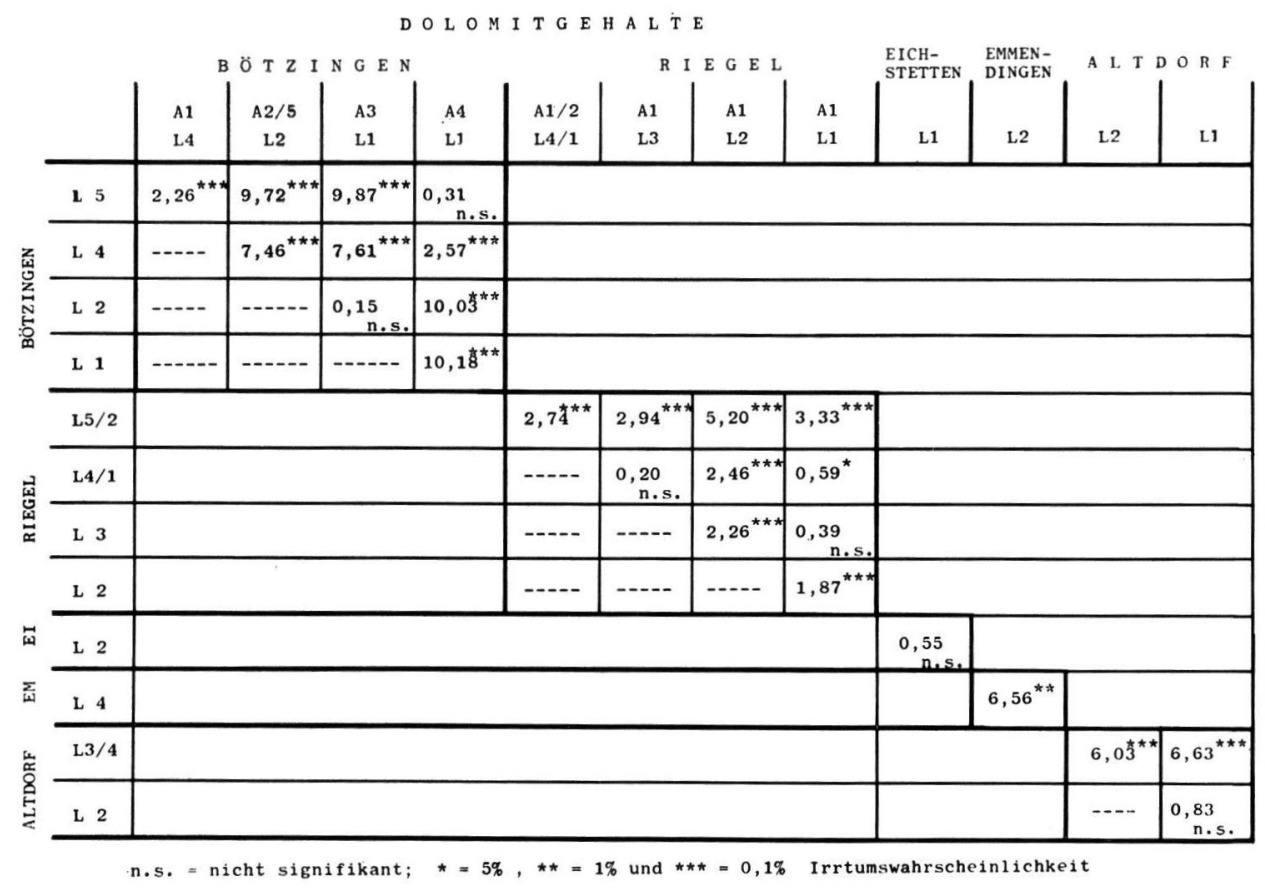

Um diesen Sachverhalt noch klarer zu demonstrieren, wurden die Lösse in Abb. 8 nicht mehr nach Aufschlüssen, sondern nach Altersgruppen geordnet. Schwarz ausgefüllte Kreise (Nichtsignifikanz) erkennen wir beim Vergleich aller Jüngeren Lösse untereinander. Daraus wird ersichtlich, daß diese nicht nur gleichaltrig, sondern stofflich besonders homogen aufgebaut sind. Die Jüngeren Lösse unterscheiden sich bis auf die Ausnahme bei Eichstetten L1/L2 von den Mittleren und Älteren Lössen signifikant; sie gehören zeitlich und stofflich nicht zusammen.

Beim Vergleich der Mittleren und Älteren Lösse untereinander findet man weniger häufig die theoretisch zu erwartende Nichtsignifikanz. M. E. liegt das nicht so sehr an der stärkeren stofflichen Differenzierung des untersuchten Materials. Der Streurahmen innerhalb der Mittleren und Älteren Lösse ist einfach größer. Natürlich könnte das „Signifikanzbild“ noch geändert werden, wenn die Irrtumswahrscheinlichkeit kleiner gewählt würde. Dies ist jedoch bei geowissenschaftlichen Untersuchungen nicht üblich. Störend in dem Bild (Abb. 8) wirken nicht so sehr die signifikanten Unterschiede zwischen gleichaltrigen Lössen (offene Sechsecke) als vielmehr die vier Fälle nichtsignifikanter Beziehungen unterschiedlich alter Lösse (Eichstetten L1/L2 und Riegel L1-L3). Wie bereits für Riegel erwähnt, kann dieses Verhalten noch nicht geklärt werden.

\subsubsection{Beziehungen zwischen Dolomit- und Gesamtcarbonatgehalt}

Aufgrund der unterschiedlichen Löslichkeit von Calcit und Dolomit war nicht zu erwarten, daß sich das ursprüngliche, d. h. genetisch vorgegebene Dolomit-Gesamtcarbonat-Verhältnis bis heute bewahrt hat. Syn- und postgenetische Carbonatlösungen und -Verlagerungen, z. T. im Zuge der Pedogenese der Paläoböden, mögen das ursprüngliche 


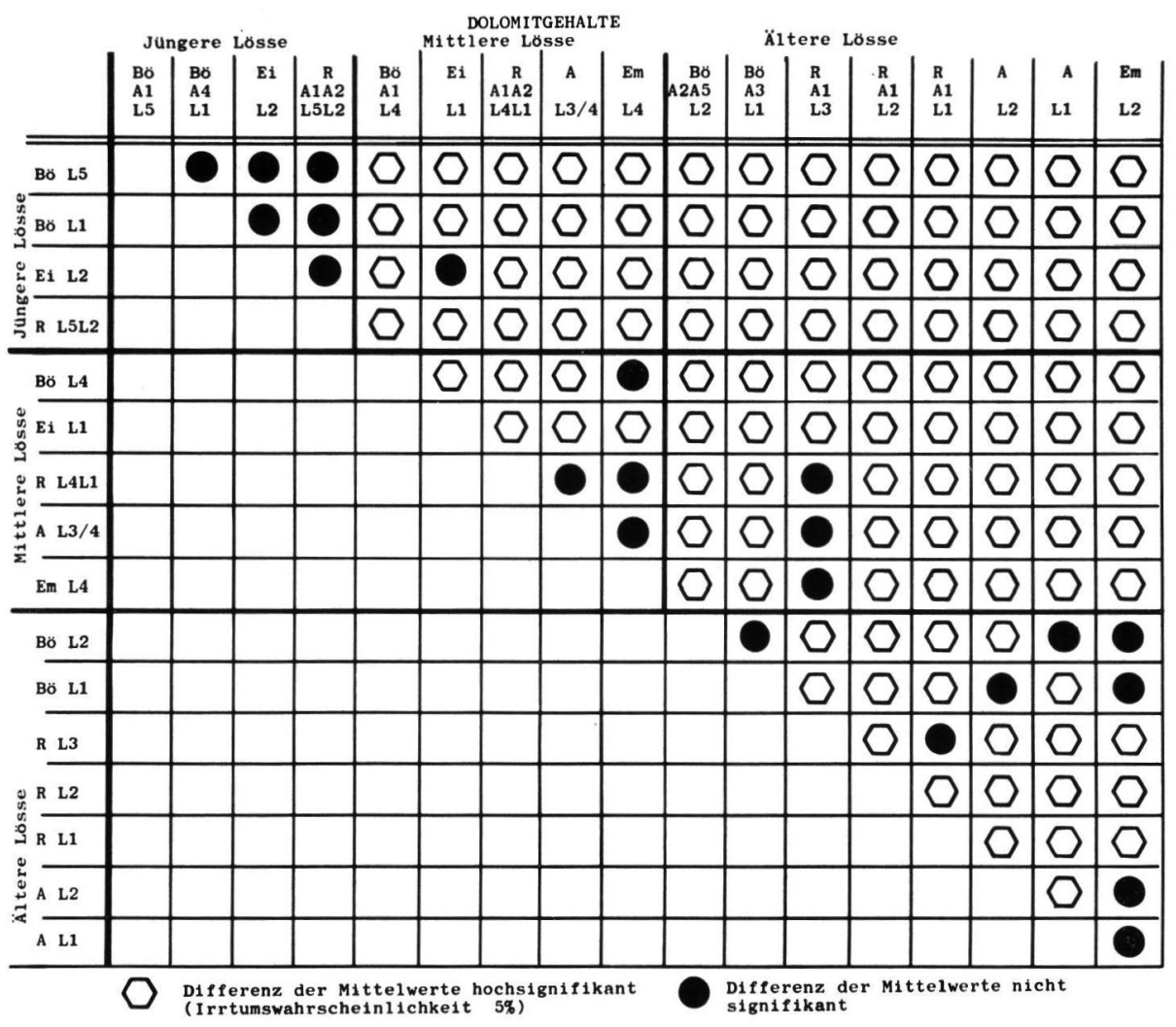

Abb. 8. Vergleich der Mittelwerte aller Lösse im Signifikanztest (gegliedert nach Altersgruppen).

Verteilungsmuster verändert haben. Um festzustellen, ob die Dolomitabnahme mit zunehmendem Alter der Lösse auch mit einer Abnahme der Gesamtcarbonatgehalte einhergeht oder nicht, wurde für jede einzelne Probe der Anteil von Dolomit in \% vom Gesamtcarbonat berechnet. Diese „Relativ-Prozente“ wurden für jeden Löß gemittelt, die Streuung und die Variationskoeffizienten berechnet. Die entsprechenden Daten sind in Tab. 2 rechts dargestellt Wir erkennen, daß diese Relativ-Prozente mit zunehmendem Alter der Lösse von ca. 34, über 29 auf $15 \%$ abnehmen und sich daher ähnlich wie die Dolomitgehalte verhalten. Dieser Trend kommt auch deutlich in der Häufigkeitsverteilung der Relativ-Prozente zum Ausdruck (Abb. 9).

Der diagnostische Wert dieser Daten für eine Differenzierung der Lösse ist sicher nicht größer als der der Dolomitdaten in Tab. 2, weswegen nicht weiter damit argumentiert werden soll. Es ist jedoch nicht zu leugnen, daß diese Relativ-Prozente ein wichtiges Kriterium für das Ausmaß sekundärer Carbonatausscheidung in den Lössen ist; denn die Abnahme der Dolomitgehalte mit zunehmendem Lößalter machen die Gesamtcarbonatgehalte aufgrund der sekundären Calcitfällung ja bekanntlich nicht im selben Maße mit, so daß die Relativ-Prozente mit zunehmendem Lößalter deutlich sinken müssen (vgl. auch Abschnitt 5.). 

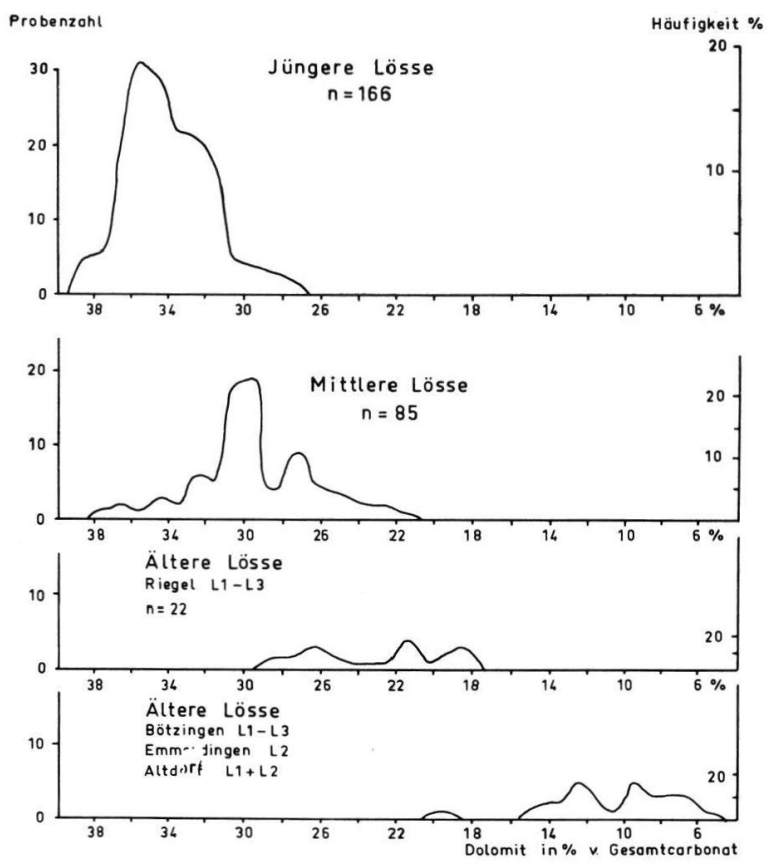

Abb. 9. Häufigkeitsverteilung, Dolomit in \% vom Gesamtcarbonat, für die Lösse.

\subsection{Die Dolomitgehaltevon Einzelproben}

In Abbildung 6 und Tabelle 2 sind auch Ergebnisse von Einzelproben aufgeführt. Es handelt sich hierbei um Lößproben, die unmittelbar unterhalb der Verlehmungsfront rezenter Parabraunerden aus der Emmendinger Vorbergzone (HäDRICH 1966) und der Teninger Fläche (FrESLE 1969) entnommen waren.

Die Dolomitgehalte der vier Einzelproben aus der Emmendinger Vorbergzone liegen im Mittel bei nur 10,9\%. Damit ist es unsicher, ob es sich hierbei um Jüngeren oder Mittleren Löß handelt. Berücksichtigt man aber die Relativ-Prozente (Tab. 2 rechts), dann wird ersichtlich, daß der Dolomitgehalt 35,6\% des Gesamtcarbonats ausmacht - ein Hinweis dafür, daß es sich doch um Jüngeren Löß handeln muß. Der Gesamtcarbonatgehalt ist unmittelbar unterhalb der Verlehmungsfront durch Lösungsverwitterung wohl z. T. schon vermindert, während der Dolomitgehalt noch relativ hoch geblieben ist.

Besonders interessant ist die $\mathrm{Probe}$ von der Teninger Flä che, einem um wenige Meter das holozäne Auenniveau der nördlichen Freiburger Bucht überragenden höheren und damit relativ früh trockengefallenen Rest der Niederterrasse. Hier lagert auf einer Fläche von 3,24 km² jüngster, spätglazialer Löß bis zu einer Mächtigkeit von 6,25 m (nach Fresle 1969), von mehr als 7,0 m nach Aussagen eines ortsansässigen Landwirtes. Die einzige bisher von mir untersuchte Probe aus einer Position unmittelbar unterhalb der dort bis $\mathrm{zu}$ einer Tiefe von $90 \mathrm{~cm}$ entwickelten Parabraunerde erbrachte einen Dolomitgehalt von $11,6 \%$ bei einem Gesamtcarbonatgehalt von 33,9\%. Das Relativ-Prozent liegt hier bei $34,2 \%$. Nicht nur die Höhe dieser Werte, sondern vor allem die topographische Position auf höherem Niederterrassenniveau sprechen eindeutig für würmzeitlichen und damit Jüngeren Löß. MüNZING (1973) stuft diesen Löß ins J u n g w ü r m ein, im 
Sinne von WoldSTEDT (1962). Solche Lößvorkommen auf Niederterrasse gibt es in der Freiburger Bucht und im Umkreis des Kaiserstuhls noch einige mehr (SCHREINER 1958). Bei allen diesen kleinen Lößvorkommen handelt es sich um den einzigen, mir bisher bekannten Fall, daß ein Löß aufgrund seiner Position auf einer Pleistozänterrasse im südlichen Oberrheingebiet überhaupt zeitlich fixiert werden kann.

4.5. Zur Anwendbarkeit der Methode der Carbonatfraktionierung bei der Lößparallelisierung

Das Hauptanliegen solcher Untersuchungen zur Carbonatfraktionierung ist, altersspezifische Kriterien zu finden, damit Lösse, deren stratigraphische Position nicht zweifelsfrei ist, einander zugeordnet werden können. Wie wir im Falle der Einzelproben gesehen haben, konnten die betreffenden Lösse über ihren charakteristischen Dolomitgehalt bzw. durch ihre Relativ-Prozente einwandfrei den Jüngeren Lössen der untersuchten Aufschlïsse zeitlich zugeordnet werden.

Ich möchte hier ein weiteres Beispiel anführen, wie die Methode der Carbonatfraktionierung helfen kann, profilmorphologisch unabhängig erscheinende Straten als lithologisch und damit auch chronologisch zusammengehörig zu erkennen:

Im Aufschluß 1 von Riegel a. K. trägt der oberste Paläobodenrest vom Typ einer Parabraunerde nicht direkt Jüngeren Löß, sondern es schaltet sich ein etwa $110 \mathrm{~cm}$ mächtiger Geröllhorizont ein. Dieser Geröllhorizont besteht aus aufgearbeiteten Lößkindeln, die etwa 60-70\% der Masse ausmachen und in Löß eingebettet sind (Abb. 10).

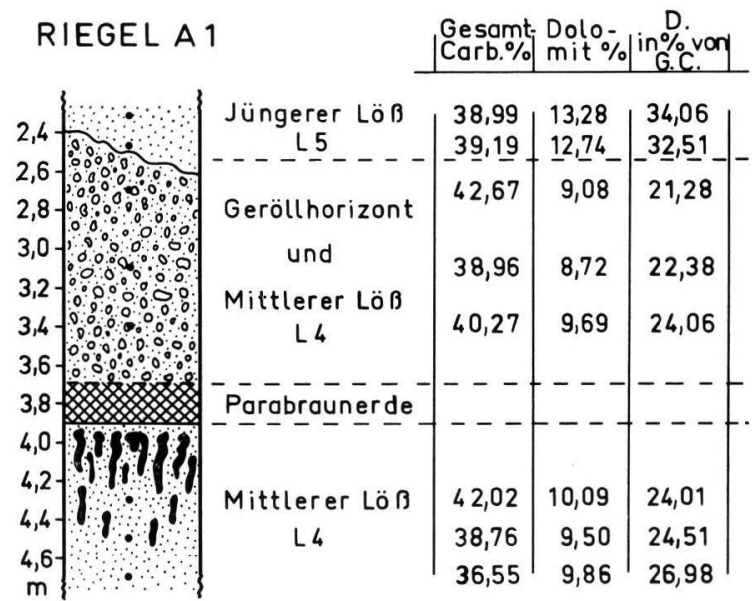

Abb. 10. Beispiel für die Zuordnung von Lössen mit Hilfe der Carbonatfraktionierung.

Profilmorphologisch war nicht sicher zu entscheiden, ob der zwischen die Gerölle eingelagerte Löß genetisch zum Jüngeren Löß im Hangenden (L 5) oder zum Mittleren Löß im Liegenden (L 4) des Paläobodenrestes gehört. Die Dolomitgehaltsbestimmung und die Berechnung der Dolomitgehalte in Prozent vom Gesamtcarbonat konnten einwandfrei klären, daß der Löß zum Mittleren Löß (L 4) dieses Aufschlusses gehört. Löß und Kindelgerölle stammen aus höherer Hangposition, wo der offensichtlich rißwürmzeitliche Paläoboden völlig abgetragen zu sein scheint und sogar ein Teil des liegenden Kindelhorizontes mit weggeräumt wurde. 


\section{Die Deutung der Ergebnisse}

Die Ergebnisse der Carbonatfraktionierung, wie sie in Abschn. 4 dargestellt wurden, bedürfen $\mathrm{m}$. E. einer Erklärung. Es ist die Frage zu stellen, ob die charakteristische Tiefenfunktion der Dolomitgehalte 1 ößgenetis ch vorgegeben war oder postge$\mathrm{n}$ e $\mathrm{t}$ is $\mathrm{ch}$ e n Veränderungen im Carbonatregime zu verdanken ist.

Betrachten wir zunächst die Tiefenfunktion des Gesamtcarbonats und sehen diese im Lichte der morphologisch überall in den Profilen - besonders deutlich aber in den Mittleren und Alteren Lössen - zu erkennenden sekundären Calcitfällungen in Form von Pseudomycelien, Bieloglaski und Lößkindeln, dann muß das Gesamtcarbonat postgenetisch entsprechenden Veränderungen unterworfen gewesen sein, deren Richtung und Größenordnung heute allerdings kaum mehr feststellbar sind, weil sie in den Analysendaten kaum aufscheinen.

Diese Veränderungen bestanden beim Jüngeren Löß offensichtlich nur in einer lokal begrenzten Umlösung der Carbonate und einer Calcitfällung, nicht aber in starken $\mathrm{Hy}$ drogencarbonat-Infiltrationen aus den Hangendpartien. Dieses Bild steht mit der Tatsache in Einklang, daß die Jüngeren Lösse der untersuchten Profile ausnahmslos Pararendzinen mit noch relativ hohen Carbonatgehalten bis zur Oberfläche tragen.

Da nun im Jüngeren Löß Anzeichen stärkerer Carbonatfällungen zurücktreten — Lößkindel gibt es nur wenige, und diese sind meist von geringer Größe sowie vereinzelt ziemlich regellos im Jüngeren Löß verteilt - muß daraus gefolgert werden, daß die holozänen Böden nie den Entwicklungsgrad der warmzeitlichen Pleistozänböden erreichten (Entkalkung, Verwitterung, Tonbildung, Entwicklungstiefe), wenn sie sich auch unter ungestörten Verhältnissen über das Pararendzinastadium hinaus entwickelt haben mögen (HÄDRICH 1966, BRONGER 1969).

Die Mittleren und die Alteren Lösse haben in den Warmzeiten des Pleistozäns eine länger andauernde und intensivere Pedogenese über sich ergehen lassen müssen, während der sich jeweils mächtige, carbonatfreie Paläoböden entwickelten. Im Zuge dieser Pedogenese wurden die jeweils liegenden Lösse in starkem Maße mit Calcit angereichert.

Wie erklären sich aber nun die Tiefenfunktionen der Dolomitgehalte? Wir halten an dieser Stelle noch einmal fest: Die Dolomitgehalte zeigen innerhalb der Lößpakete keine tendenzmäßige Veränderung in der Tiefenfunktion; diese erfolgt vielmehr beim Übergang zum nächstälteren bzw. nächstjüngeren Löß sprungartig, so daß ein Treppenprofil der Tiefenfunktion vorliegt.

Aus der Kenntnis der Dolomitgenese heraus dürfen wir wohl davon ausgehen, daß der in den obersten Lößabschnitten im Zuge der Pedogenese gelöste Dolomit abwärts nicht wieder als solcher ausgefällt wird. In tieferen Profilabschnitten vorhandener Dolomit kann daher mengenmäßig entweder nur e r h a l t e n od e r ver minder t worden sein, entweder relativ, durch Zufuhr von Calcit oder absolut, durch bevorzugte Dolomitlösung.

\subsection{Zur relativen Verminderung des Dolomitgehaltes}

Das Argument der relativen Verminderung des Dolomitanteiles durch Calcitzufuhr aus dem Hangenden entfällt als alleinige Erklärung für die heutigen Verhältnisse, wenn man davon ausgeht, daß am Dolomit selbst seit der Lößablagerung keine Verwitterung stattgefunden hat, da die Calcitzufuhr niemals das Ausmaß erreichte, um die Tiefenfunktionen des Dolomitgehaltes zu erklären. 


\subsection{Zur absoluten Verminderung des Dolomitgehaltes}

Im Falle einer absoluten Verminderung des Dolomitgehaltes müßte es zu einer postgenetischen bevorzugten Dolomitverwitterung gekommen sein. Es gibt sicher mehrere Argumente und Erkenntnisse dafür, daß eine postgenetische spezifische Dolomitverwitterung ausscheidet. Ich möchte im folgenden nur auf Ergebnisse von FEZER (1969) und auf einen eigenen Modellversuch zur Carbonatlösung eingehen.

\subsubsection{Zur „Tiefenverwitterung“ des Lösses}

FEZER (1969) hat in Schotterprofilen im Alpenvorland eine sog. Tiefenverwitterung festgestellt, die er vor allem an der Zermürbung der Dolomitgerölle zu "Dolomitasche“ erkannte und an der er u. a. auch die Dauer der pleistozänen Warmzeiten abzuschätzen versuchte. Die Zermürbung schritt deutlich der Lösungsfront aller Carbonate voraus.

Es könnte nun jemand auf den Gedanken kommen, im Löß seien postgenetisch ähnliche Vorgänge abgelaufen. M. E. ist aber ein Vergleich mit den Ergebnissen Fezers für den Löß nicht zulässig. Fezer erklärt seine Dolomitverwitterung aus der besonderen Wasserbewegung im Schotter, aus der Porosität der Dolomitgerölle, bei denen er bis zu $14 \%$ Gesamtporenvolumen feststellte und aus der selektiven Lösung geringer Mengen der $\mathrm{CaCO}_{3}-\mathrm{Komponente} \mathrm{in} \mathrm{den} \mathrm{Dolomitgeröllen,} \mathrm{wonach} \mathrm{die} \mathrm{Dolomitstruktur} \mathrm{rasch} \mathrm{zusam-}$ menbräche. Darüber, was danach mit der Dolomitasche passiert, sagt FEZER m. W. nichts aus.

Im Löß haben wir es im Gegensatz zu Geröllen überwiegend mit Einzelmineralen zu tun, bei denen man von der Zermürbung der Struktur und von Porosität nicht mehr sprechen kann. Auch die Wasserbewegung im Löß ist eine völlig andere als im Schotter. Nach vorläufigen Ergebnissen aus noch unveröffentlichten hydrologischen Untersuchungen des Geographischen Instituts I der Universität Freiburg i. Br. ${ }^{7}$ ) im Löß des Ostkaiserstuhls ist bei Löß eine mittlere Sickergeschwindigkeit von $1 \mathrm{~m} / \mathrm{J} a h r$ anzunehmen. Bei solch geringer Geschwindigkeit muß sich die Bodenlösung schon beim Durchlaufen der obersten Dezimeter des Lösses im Wechselspiel eines sich dort ständig verändernden $\mathrm{CO}_{2}$-Partialdruckes bis zu einem Gleichgewicht mit Hydrogencarbonat sättigen, so daß in größeren Profiltiefen, die immerfeucht, gleichmäßig temperiert und ohne nennenswerte Durchwurzelung sind, aufgrund konstanten $\mathrm{CO}_{2}$-Partialdruckes keine weitere Lösungsaktivität mehr denkbar ist.

Eine selektive Dolomitlösung aufgrund leicht höherer Löslichkeit von Dolomit bei niedrigem $\mathrm{CO}_{2}$-Partialdruck gegenüber Calcit, wie sie FEzer für die Schotter annahm, dürfte im Löß kaum zu dieser Dolomitverteilung geführt haben, sonst hätte sich das analytisch aus der Tiefenfunktion des Dolomits ablesen lassen müssen.

Eine bevorzugte Dolomitverwitterung müßte in höheren Profilteilen eingesetzt haben, so daß die Dolomitgehalte in einem Lößpaket heute von oben nach unten zunehmen müßten. Es ist aber nichts dergleichen festzustellen.

\subsubsection{Modellversuch zur Carbonatlösung}

Um zu klären, ob durch perkolierendes Sickerwasser im Löß eine spezifische Dolomitlösung im Sinne einer absoluten Dolomitverminderung erfolgt, habe ich in der Zeit vom 7. 3. bis zum 7. 5. 1975 im Labor einen Carbonatlösungsversuch durchgeführt.

$\mathrm{Zu}$ diesem $\mathrm{Z}$ wecke wurde dolomitreiches Lößmaterial aus der Tiefe 6,9-7,1 $\mathrm{m}$ des Aufschlusses A 4 von Bötzingen (Jüngerer Löß) in Säulen von $6 \mathrm{~cm}$ Innendurchmesser eingefüllt und durch leichtes Einstampfen auf ca. $40 \mathrm{~cm}$ Füllhöhe gebracht. Die Lößfüllung war jeweils oben und unten

7) Freundliche mündliche Mitteilung der Herren G. Luft und G. Mongenschweis.

8 Eiszeitalter u. Gegenwart 
durch eine ca. $2 \mathrm{~cm}$ dicke Quarzsandschicht abgeschlossen. Die Säulen besaßen einen Boden aus Glasfrittenmaterial und wurden so auf Nutschen mit Saugflasche montiert, daß jederzeit ein geringer Unterdruck von etwa $300-500 \mathrm{~cm}$ WS angelegt werden konnte. Der Versuch wurde in einfacher Wiederholung durchgeführt.

Zunächst wurde der Löß über einen Zeitraum von ca. 24 Stunden völlig gesättigt, dann wurde ein Unterdruck von etwa $400 \mathrm{~cm}$ WS angelegt, um überschüssiges Wasser zu entfernen und um die Funktionsfähigkeit der Anlage zu prüfen. Danach wurden die beiden Säulen im Abstand von 1-25 Tagen - die Verweilzeit wurde absichtlich variiert - mit je $50 \mathrm{ml}$ aqua dest. perkoliert, das zuvor bei +5 bis $+6^{\circ} \mathrm{C}$ im Kühlschrank aufbewahrt und danach ca. 1 Stunde lang unter weiterer Kühlung mit $\mathrm{CO}_{2}$ angereichert worden war. Das frisch aufgegebene Perkolationswasser besaß eine Temperatur von +6 bis $+9^{\circ} \mathrm{C}$. Nach etwa 3-4 Stunden war unter dem Einfluß des angelegten und halbstündlich kontrollierten Unterdruckes gewöhnlich die Perkolation zum Stillstand gekommen. Das gewonnene Filtrat wurde mit $20 \mathrm{ml} 10 \%$ iger $\mathrm{HCl}$ aufgenommen, in Polyäthylenflaschen überführt und bis zur Messung im Kühlschrank aufbewahrt. Im Labor herrschten zu keiner Zeit höhere Temperaturen als $15^{\circ} \mathrm{C}$, so daß für eine Carbonatlösung relativ optimale Bedingungen vorlagen. Nach Abschluß des Versuches kamen die Proben zur Messung im AAS, wobei die Konzentration von $\mathrm{Ca}$ und $\mathrm{Mg}$ festgestellt wurde. Für jede Probe wurde das Konzentrationsverhältnis $\mathrm{Ca}$ zu $\mathrm{Mg}$ gebildet und die Verhältnisse gemittelt.

Das Ergebnis des Carbonatlösungsversuchs ist in Tabelle 4 dargestellt. Das $\mathrm{Ca}: \mathrm{Mg}$ Verhältnis belief sich im Mittel der 15 Perkolationen auf 8,3315.

Vergleichsweise betrug das Mittel $\left(\bar{x}_{5}\right)$ der Verhältnisse für die beiden Kationen bei 5 untersuchten Proben aus der Tiefe 6,7-7,3 m des Aufschlusses A 4 von Bötzingen bei vollständiger Carbonatlösung ( $\mathrm{HCl} / \mathrm{AAS}-M e t h o d e)$ 7,2060, das entsprechende Mittel $\left(\bar{x}_{162}\right)$ für alle untersuchten Jüngeren Lösse 7,5746.

Wenn also Calcit und Dolomit im Verhältnis ihrer Gehalte im Löß gelöst worden wären, hätte der Säulenversuch ein Verhältnis bringen müssen, das dem der HCl/AASMessung für den gleichen Löß entspräche, also 7.2. Wenn Dolomit bevorzugt gelöst worden wäre, dann hätte das Verhältnis noch kleiner sein müssen, also $\langle 7,0$. Das gefundene Verhältnis von im Mittel 8,3315 bestätigte voll die Vermutung, daß im Löß eine bevorzugte Dolomitlösung nicht erfolgt. Wenn dies schon in den nur knapp $40 \mathrm{~cm}$ hohen Lößsäulen nicht der Fall ist, wo das Perkolationswasser aufgrund des hohen $\mathrm{CO}_{2}$-Partialdruckes eine stark carbonatlösende Kraft besitzt, wieviel weniger kann das in Natur in größerer Lößtiefe passieren!

\section{Tabelle 4}

Ergebnisse eines Modellversuches zur Carbonatlösung unter Laborbedingungen

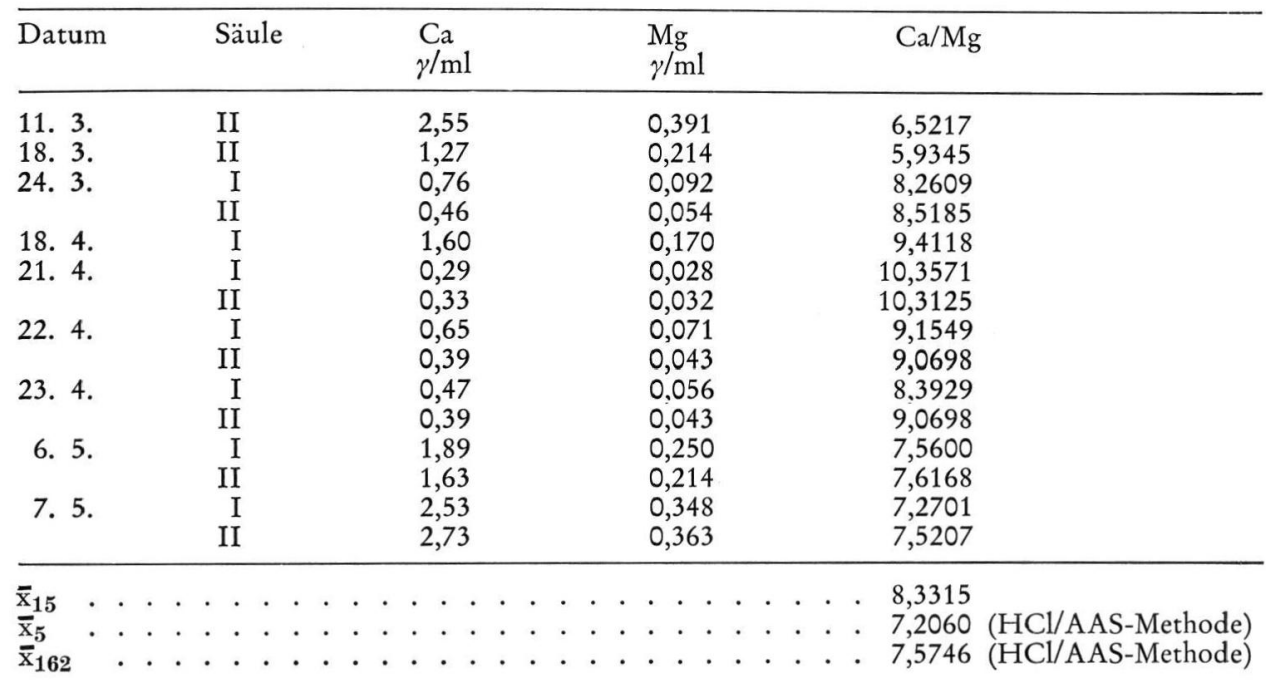


5.3. Zur Möglichkeit der Kombination von absoluter Verminderung des Gesamtcarbongehaltes und nachfolgender relativer Verminderung des Dolomitgehaltes

Wie aus Abschnitt 5.2.2. ersichtlich, halte ich eine absolute Verminderung des Dolomitgehaltes durch selektive Dolomitlösung für unwahrscheinlich. Es wäre aber zumindest theoretisch denkbar, daß der Löß postgenetisch, noch während der Kaltzeit, in der er gebildet wurde, durch Carbonatlösung und Abfuhr in den Untergrund, ins Grundwasser im Sinne des von mir ermittelten Kationenverhältnisses von 8,33 doch einen Teil seines Gesamtcarbonats absolut eingebüßt hätte, womit auch der Dolomitanteil vermindert worden wäre. Der heute ermittelte hohe Gesamtcarbonatgehalt könnte dann der sekundären Calcitfällung aus der hangenden Pedosphäre während der nachfolgenden Warmzeiten zu verdanken sein.

Gegen diese Auffassung spricht folgende Überlegung: Die Gesamtcarbonatlösung hätte aufgrund der oben in einem Lößpaket höheren Lösungsbereitschaft des Sickerwassers stärker sein und abwärts allmählich aufhören müssen. Der Dolomit, da er nicht durch sekundäre Fällung aus höheren Profilteilen hat ergänzt werden können, hätte oben weniger, weiter abwärts stärker erhalten bleiben müssen, während das mögliche Calcitdefizit durch sekundäre Fällung später hätte wieder ausgeglichen werden können.

Leider zeigt aber die Tiefenfunktion der Dolomitgehalte innerhalb eines Lößpaketes keine Zunahme von oben nach unten. Dies wäre nur dann durch die o. g. Theorie zu erklären, wenn eine Gesamtcarbonat- und Dolomitlösung so gleichmäßig über eine Tiefe von z. T. mehr als $10 \mathrm{~m}$ erfolgen könnte, und das halte ich für ausgeschlossen.

Die Gleichmäßigkeit der Dolomittiefenfunktion und die deutlichen Sprünge beim Übergang in ein anderes Lößpaket müssen daher anders zu erklären sein.

\section{4. Über die Möglichkeit der Erhaltung des ursprünglichen Dolomitanteiles - Anlieferungshypothese}

Wenn auch eine geringe relative Verminderung des Dolomitanteiles durch sekundäre Calcitzufuhr aus dem Hangenden der Lößpakete im Sinne von Abschn. 5.1. nicht ganz ausgeschlossen werden kann, kommt $\mathrm{m}$. E. doch der Erhaltung der mit der Lößablagerung vorgegebenen Dolomitgehalte die größere Wahrscheinlichkeit zu. Wir müßten dann allerdings nach einem Mechanismus suchen, der den Zusammenhang mit der Lößgenese erhellt. Die sog. Anlieferungshypothese läßt zumindest theoretisch zwei Erklärungsmöglichkeiten zu:

\subsubsection{Hypothese einer direkten Anlieferung}

Diese Hypothese geht davon aus, daß während der verschiedenen Kaltzeiten des Pleistozäns im Hauptabtragungsgebiet, den Alpen, Gesteine mit zunehmendem Dolomitgehalt an der Oberfläche anstanden, so daß über die glazigenen und glazifluvialen Sedimente, sozusagen auf direktem Wege und ohne postgenetische Veränderungen, den jeweiligen Lössen ganz bestimmte Dolomit-Gesamtcarbonatverhältnisse aufgeprägt worden seien, so wie wir sie heute feststellen. Ich halte diese Möglichkeit für unwahrscheinlich, da sie durch nichts zu beweisen ist.

\subsubsection{Hypothese der Anlieferung nach prägenetischer Dolomitanreicherung}

Viel mehr Wahrscheinlichkeit besitzt die Hypothese der Dolomitanlieferung nach prägenetischer Anreicherung, wobei der Begriff „prägenetisch“ auf die Lößgenese zielt. 
Diese Hypothese geht davon aus, daß in den glazialen und glazifluvialen Sedimenten aller Altersstufen zu allen Zeit im Alpenvorland im Sinne von FEzER (1969) eine Aufbereitung der Dolomitgerölle zu Dolomitsand und -schluff stattfand. Dies führte jeweils in den - bezogen auf eine sich anbahnende neue Vereisung und damit neue Lößbildung - voraufgegangenen Ablagerungen zur absoluten Anreicherung äolisch verwertbarer Dolomitpartikel. Die absolute Anreicherung bezieht sich auf den Ausgangsgehalt an feinen Dolomitpartikeln zum Zeitpunkt der zu betrachtenden Ablagerung. Beim jeweils folgenden Gletschervorstoß wurde das so angereicherte Material entweder direkt vom Gletschereis oder von seinen Schmelzwässern umgelagert und in die der neuen Vereisung zugehörigen Sedimente inkorporiert, wobei der Dolomitgehalt in der Sand- und Schlufffraktion gegenüber dem des direkt aus den Alpen herantransportierten Materialanteiles anstieg.

So mußten die Pleistozänsedimente im Alpenvorland aber auch in der Oberrheinebene, als dem Hauptausblasungsgebiet der oberrheinischen Lösse, ständig dolomitreicher werden, weil es bei jeder neuen Vereisung zum Ablauf des gleichen Mechanismus kam.

Auf diese Weise konnten die Lösse immer dolomitreicher werden, obwohl die jeweils im Hauptabtragungsgebiet bereitgestellten neuen Sedimente im Sinne von Abschn. 5.4.1. primär nicht dolomitreicher zu sein brauchten.

Diese Hypothese wird gestützt durch zwei Erkenntnisse bzw. Befunde:

1. Vom Alteren zum Mittleren Löß erfolgt der größte Dolomitsprung nach oben. Den Mittleren Löß stelle ich in die Rißeiszeit. So benötige ich für die Rißeiszeit ${ }^{8}$ ) ein außergewöhnliches Maß an Aufarbeitung und Dolomitanreicherung in älterem Material, das vom Rißgletscher durch seinen weiten Vorstoß fast vollständig überfahren und damit weitgehend in die Rißsedimente aufgenommen wurde. Dieses ältere Material wurde im Rheingletscherbereich, dem der oberrheinische Löß wohl zum größten Teil entstammt, durch den am weitesten vorstoßenden Mindelgletscher im Sinne von Graul (1968) bereitgestellt, das während der relativ langdauernden Mindel/Riß-Warmzeit einer besonders tiefgreifenden Dolomitverwitterung im Sinne FEzERs und damit Dolomitanreicherung in den feinen Fraktionen unterlegen haben dürfte.

2. Aus den Ergebnissen der Untersuchungen von FEzer (1969) ist abzuleiten, daß im Mittel der Schluff von Würmschottern mit ca. 1:600 ein engeres $\mathrm{Ca}: \mathrm{Mg}$ - Verhältnis aufweist als der von Günzschottern, das bei ca. 1 : 1200 liegt. Dieses Ergebnis muß nicht zwangsläufig eine bevorzugte Schluffverwitterung im Sinne von „je älter der Schluff, um so mehr an Dolomit verarmt" bedeuten; sie kann auch im Sinne meiner Hypothese der Dolomitanlieferung nach prägenetischer Dolomitanreicherung gedeutet werden und lauten: "Je jünger das Sediment, um so mehr Dolomit durch Anreicherung im Schluff.“

Wenn letztere Erscheinung schon für unbedeckte Terrassenschotter gilt, wieviel mehr muß das dann für den Schluff in Lößprofilen zutreffen, wo aufgrund wiederholter Sedimentüberdeckungen im Vergleich zu unbedeckten Schotterkörpern keine über große Teile des Pleistozäns hin am gleichen Löß fortwirkende Tiefenverwitterung möglich erscheint?

\section{Schriften}

Bibus, E.: Ausbildung und Lagerungsverhältnisse quartärer Tuffvorkommen in der Wetterau. Notizbl. hess. L.-Amt Bodenforsch. 101, 346-361, Wiesbaden 1973.

Bronger, A.: Lösse, ihre Verbraunungszonen und fossilen Böden. Ein Beitrag zur Stratigraphie des oberen Pleistozäns in Südbaden. - Schriftenr. Geogr. Inst. Univ. Kiel 14, 2, 113 S., Kiel 1966.

- : Zur Klimageschichte des Quartärs von Südbaden auf bodengeographischer Grundlage. Peterm. Geogr. Mitt., 113. Jg., 2, 112-124, Gotha 1969.

8) Im Sinne von Graul (1968). 
Bronger, A. \& Hädrich, F.: Le Loess du Sud-Ouest de L'Allemagne. - Suppl. au Bull. de l'Ass. Franc. pour l'Etude du Quarternaire, VIIIe Congr. INQUA, 23-27, Paris 1969.

BRunNACKER, K.: Beiträge zur Stratigraphie des Quartärprofils von Kärlich am Mittelrhein. - Mz. Naturw. Arch., 10, 77-100, Mainz 1971.

FEzer, F.: Tiefenverwitterung circumalpiner Pleistozänschotter. - Heidelb. Geogr. Arb., H. 24, 144 S., Heidelberg 1969.

FrESLE, F.: Zur Genese der Lößinseln auf den würmzeitlichen Schwemmfächern von Dreisam und Elz (nördliche Freiburger Bucht). - Unveröff. maschinenschr. Diss. Freiburg i. Br. 1969.

Graul, H.: Führer zur zweitägigen Exkursion im nördlichen Rheingletschergebiet, August 1968, S. 31-75 in: Beiträge zu den Exkursionen anläßlich der DEUQUA-Tagung August 1968 in Biberach an der Riß. - Heidelb. Geogr. Arb., H. 20, 124 S., Heidelberg 1968.

Guenther, E. W.: Sedimentpetrographische Untersuchung von Lössen. Teil I: Methodische Grundlagen mit Erläuterung an Profilen. - Fundamenta, Monographien zur Urgeschichte, Reihe B, 1, 91 S., Köln und Graz 1961.

HäDrich, F.: Die Böden der Emmendinger Vorbergzone (Südliches Oberrheingebiet). - Ber. Naturf. Ges. Freiburg i. Br. 56, 23-76, Freiburg 1966.

- : Zur Anwendbarkeit einiger bodenkundlicher Untersuchungsmethoden in der paläopedologischen und quartär-geologischen Forschung unter besonderer Berücksichtigung der Untersuchung von Proben aus Lößaufschlüssen. - Ber. Naturf. Ges. Freiburg i. Br. 60, 103-137, Freiburg 1970.

KHodary-Erssa, O.: Feinstratigraphische und pedologische Untersuchungen an Lößaufschlüssen im Kaiserstuhl (Südbaden). - Freibg. Bodenkundl. Abh. 2, 149 S., Freiburg 1968.

MülLER, G.: Methoden der Sedimentuntersuchung; Sedimentpetrologie, Teil I, E. Schweizerbart'sche Verlagsbuchhandlung, Stuttgart 1964.

MüNZING, K.: Beiträge zur quartären Molluskenfauna Baden-Württembergs. - Jh. geol. Landesamt Bad.-Württ 15, 161-185, Freiburg i. Br. 1973.

Noммік, H.: An examination of procedures for determining carbonates in soils and related materials. - Comm. in Soils Sci. and Plant Anal., Vol. 5, 4, 319-330, 1974.

PAEPE, R.: Les unites litho-stratigraphiques du pleistocene superieur de la Belgique. - Suppl. au Bull. de l'Ass. Franc. pour l'Etude du Quarternaire. VIIIle Congr. INQUA, 45-51. Paris 1969.

Petersen, G. W. \& Chesters, G.: Quantitative determination of calcite and dolomite in pure carbonates and limestones. - Soil Sci. 17, 2, 317-327, 1966.

Schönhals, E., Rhodenburg, H. \& Semmel, A.: Ergebnisse neuerer Untersuchungen zur Würmlößgliederung in Hessen. - Eiszeitalter u. Gegenwart 15, 199-206, Öhringen 1964.

Schlichting, E. \& Blume, H. P.: Bodenkundliches Praktikum, 209 S., Parey Hamburg/Berlin 1966.

SchreINER, A.: Niederterrasse, Flugsand und Löß am Kaiserstuhl (Südbaden). - Mitt. Bad. Landesver. Naturkde. u. Natursch., N. F. 7, 2, 113-125, Freiburg i. Br. 1958.

Semmel, A.: Stratigraphie du loess dans les Hesses meridionales et Rhenanes. - Suppl. au Bull. de l'Ass. Franc. pour l'Etude du Quarternaire. VIIIe Congr. INQUA, 81-83, Paris 1969.

Semmel, A. et al.: Das Eiszeitalter im Rhein-Main-Gebiet. - Rhein-Mainische Forsch. 78, 215 S., Frankfurt a. M. 1974.

Woldstedt, P.: Uber die Gliederung des Quartärs und Pleistozäns. - Eiszeitalter u. Gegenwart 13, 115-124, Öhringen 1962.

Manuskript eingeg. 9. 7. 75.

Anschrift des Verf.: Dr. F. Hädrich, Inst. f. Bodenkunde u. Waldernährungslehre d. Univ. Freiburg, 78 Freiburg i. Br., Bertoldstraße 17. 